Page 1 of 45. (C) The Author(s), 2020. Published by Cambridge University Press on behalf of Royal Aeronautical Society

doi:10.1017/aer.2020.124

\title{
An estimation method for the fuel burn and other performance characteristics of civil transport aircraft during cruise: part 2, determining the aircraft's characteristic parameters
}

\author{
D.I.A. PolliD \\ d.i.a.poll@cranfield.ac.uk \\ Emeritus Professor of Aerospace Engineering \\ Cranfield University
}

\section{U. Schumann}

Deutsches Zentrum für Luft- und Raumfahrt

Institut für Physik der Atmosphäre

Oberpaffenhofen

Germany

\section{ABSTRACT}

A simple yet physically comprehensive and accurate method for the estimation of the cruise fuel burn rate of turbofan powered transport aircraft operating in a general atmosphere was developed in part 1 . The method is built on previously published work showing that suitable normalisation reduces the governing relations to a set of near-universal curves. However, to apply the method to a specific aircraft, values must be assigned to six independent parameters and the more accurate these values are the more accurate the estimates will be. Unfortunately, some of these parameters rarely appear in the public domain. Consequently, a scheme for their estimation is developed herein using basic aerodynamic theory and data correlations. In addition, the basic method is extended to provide estimates for cruise lift-to-drag ratio, engine thrust and engine overall efficiency. This step requires the introduction of two more independent parameters, increasing the total number from six to eight. An error estimate and sensitivity analysis indicates that, in the aircraft's normal operating range and using the present results, estimates of fuel burn rate are expected to be in error by no more than $5 \%$ in the majority of cases. Initial estimates of the characteristic parameters have been generated for 53 aircraft types and engine combinations and a table is provided.

Keywords: Optimum fuel burn; Minimum fuel burn; Environmental impact; Cruise emissions; Minimum fuel trajectory; Aircraft characteristics 


\section{NOMENCLATURE}

\begin{tabular}{|c|c|}
\hline$A$ & coefficient - Equations (A-5) and (A-6) \\
\hline$A_{e}$ & core and bypass jet exit cross-sectional areas summed over all engines \\
\hline$A R$ & wing aspect ratio \\
\hline$a$ & constant in the skin friction law $(=0.0269)$ - Equation $(7)$ \\
\hline$a_{\infty}$ & speed of sound $=\left(\gamma \mathfrak{R} T_{\infty}\right)^{1 / 2}$ \\
\hline$B$ & coefficient - Equations (A-5) and (A-7) \\
\hline$B P R$ & engine nominal bypass ratio \\
\hline$b$ & exponent in skin friction law $(=0.14)-$ Equation $(7)$ \\
\hline$b_{f}$ & fuselage width \\
\hline$C d$ & airframe drag coefficient $=D /\left(0.5 \gamma p_{\infty}\left(M_{\infty}\right)^{2} S_{r e f}\right)$ \\
\hline$C d_{o}$ & zero-lift drag coefficient \\
\hline$C d_{w}$ & wave drag coefficient \\
\hline$C_{L}$ & overall lift coefficient $=L /\left(0.5 \gamma p_{\infty}\left(M_{\infty}\right)^{2} S_{r e f}\right)$ \\
\hline$C_{l}$ & local lift coefficient - Equation (30) \\
\hline$C_{F}$ & mean skin friction coefficient \\
\hline$C_{t}$ & engine net thrust coefficient $=F_{n} /\left(0.5 \gamma p_{\infty}\left(M_{\infty}\right)^{2} A_{e}\right)$ \\
\hline$c$ & local wing chord measured in the streamwise direction \\
\hline$D$ & total drag force \\
\hline$E$ & coefficient - Equation (8) \\
\hline$e$ & aircraft Oswald efficiency factor \\
\hline FCOM & flight crew operating manual \\
\hline$F L$ & flight level \\
\hline$F M$ & fuel mass \\
\hline$F_{F}, F_{I}, F_{o}, F_{S}$ & $\begin{array}{l}\text { component form, interference, overall and secondary drag factors - } \\
\text { Equation (23) and Appendix B }\end{array}$ \\
\hline$F_{n}$ & net thrust, summed over all engines \\
\hline$F_{w v}$ & aircraft weight variant factor - Equation (63) \\
\hline$f_{1-7}$ & functions - Appendix A and Equations (32) to (34) \\
\hline$G_{2-7}$ & functions - Appendix A and Equation (87) \\
\hline$g$ & acceleration due to gravity $(9.80665 \mathrm{~m} / \mathrm{s}$ at sea level $)$ \\
\hline$g_{1-3}$ & functions - Equations (97) to (99) \\
\hline$k_{1}$ & miscellaneous lift-dependent drag factor - Equation (26) \\
\hline$L$ & lift force \\
\hline$L C V$ & lower calorific value of fuel $\left(\approx 43 \times 10^{6} \mathrm{~J} / \mathrm{kg}\right.$ for kerosene $)$ \\
\hline$L / D$ & lift-to-drag ratio \\
\hline$L M$ & landing mass \\
\hline$l$ & characteristic streamwise length $=S_{r e f}^{1 / 2}$ \\
\hline$M_{\infty}$ & flight Mach number $=V_{\infty} / a_{\infty}$ \\
\hline$M P M$ & maximum payload (passengers + cargo) mass $(=M Z F M-O E M)$ \\
\hline MTOM & maximum permitted take-off mass \\
\hline
\end{tabular}


MZFM maximum zero fuel mass (maximum permitted aircraft mass without fuel)

$m$

$n$

OEM

PM payload mass (passengers + cargo)

$p$

$R^{a c}$

$R_{t}$

$\Re$

$S$

$S_{\text {ref }}$

$s$

$T$

$T_{o}$

TFM

TOM

$t / c$

$V_{\infty}$

$X_{t}$

$y$

$Z F M$

$\alpha_{t}$

$\beta_{\text {min }}$

$\gamma$

$\delta_{1}$

$\delta_{2}$

$\delta_{3}$

$\varepsilon$

$\varepsilon_{c d}$

$\varepsilon_{t}$

$\eta_{o}$

$\eta_{1}, \eta_{2}$

$\iota$

$\kappa$

$\lambda$

$\lambda_{f}$

$\mu$

$v$

instantaneous total aircraft mass

ratio of $\left(\eta_{o} L / D\right)_{\text {avg }}$ to $\left(\eta_{o} L / D\right)_{o}$ without fuel)

static pressure and destination points

distance travelled through the air

wing span

static temperature

true air speed centre line. Equation (70)

induced drag imperfection factor

lift-dependent wave drag factor

overall 'lost' fuel index

constants in Equation (35)

atmospheric coefficient

coefficient - Equation (A-21)

reserve fuel factor - Equation (74)

fuselage slenderness ratio

dynamic viscosity

coefficient - Equation (A-27) aircraft operational empty mass (mass of aircraft without payload and

characteristic Reynolds number - Equation (3)

ground distance measured along the great circle containing the departure

gas constant for air $(287.05 \mathrm{~J} /(\mathrm{kg} \mathrm{K}))$

aerodynamic reference wing area (Airbus definition)

total temperature $=T\left(1+((\gamma-1) / 2) M_{\infty}^{2}\right)$

mass of the trip fuel (fuel burned between 'brakes off' at take-off and 'brakes on' at the end of the landing run)

total aircraft mass at the start of the take-off run

wing streamwise thickness-to-chord ratio

non-dimensional great circle distance - Equation (67)

spanwise coordinate - drawn normal to fuselage centre line with origin on

zero-fuel mass (mass of aircraft, including payload, but without any fuel)

ratio of trip fuel mass to aircraft take-off mass - Equation (66)

ratio of the minimum reserve fuel mass to aircraft take-off mass -

ratio of specific heats for air $(=1.4)$

induced drag wing-fuselage interference factor

function - Equations (17) and (A-19)

'lost' fuel index for both climb and descent phases

propulsion system overall efficiency - Equation (2) 


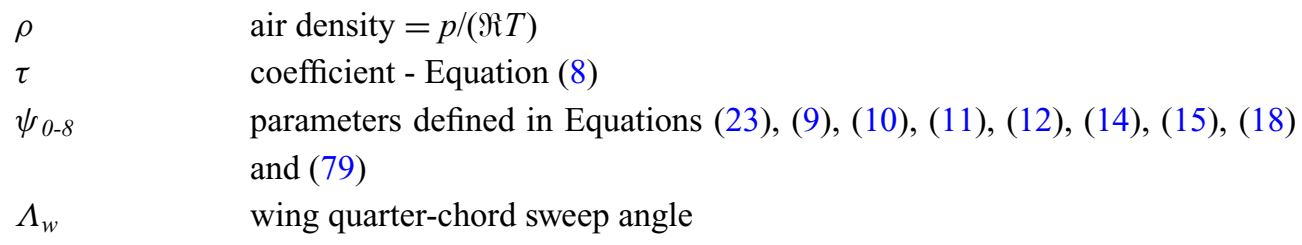

\section{Superscripts}

$\begin{array}{ll}a c & \text { whole aircraft value } \\ i & \text { sub-component value } \\ F P & \text { flat-plate value } \\ R & \text { evaluated at constant Reynolds number }\end{array}$

\section{Subscripts}

ad additional

alt alternate

avg average

$B$ best, or local maximum, value

cont contingency

ext extra

$d a \quad$ to alternate airport

$f_{c} \quad$ final cruise

fr final reserve

HS high speed

ic initial cruise

$L S \quad$ low speed

$L R C \quad$ long range cruise

$\max$ maximum value

$M R C$ maximum range cruise

$M O \quad$ maximum permitted operational value

min minimum value

nc not consumed on flight

$o \quad$ when $\left(\eta_{o} L / D\right)$ has its absolute maximum value

res reserve

ref reference

$T E \quad$ at the entry to the turbine

TP at the tropopause

$\infty \quad$ flight, or freestream, value

\subsection{INTRODUCTION}

The majority of global aviation emissions come from civil air transport, which is dominated by large, turbofan-powered aircraft. Recently, Poll ${ }^{(1)}$ and Poll and Schumann ${ }^{(2)}$ demonstrated that, by suitable normalisation, the cruise fuel burn performance of this class of aircraft can 
be described by a system of simple equations, provided that values are assigned to a set of three independent parameters. The first is the absolute maximum, or optimum, value of the product of the engine overall efficiency and the airframe lift-to-drag ratio, whilst the second and third parameters are the flight Mach number and aircraft lift coefficient at which this optimum occurs. To simplify the analysis, Poll ${ }^{(1)}$ assumed that the Reynolds number was constant everywhere. However, Poll and Schumann ${ }^{(2)}$ extended the analysis to allow the Reynolds number to vary with both speed and altitude in a general atmosphere. This extension resulted in the number of independent aircraft parameters increasing from three to six. Nevertheless, the basic simplicity of the original method was retained.

In this paper, schemes for the estimation of all the independent parameters are developed and the basic method is extended to include cruise lift-to-drag ratio, engine thrust and engine overall efficiency. These latter two quantities are particularly important since they influence contrail formation, as demonstrated by Schumann, Busen and $\mathrm{Plohr}^{(3)}$. Where possible, the problem of estimating these parameters is approached using classical aerodynamic theory and, where necessary, empirical correlations based upon data that are available in the open literature. Initial results are obtained for a range of narrow-body and wide-body transport aircraft and some business jets.

The complete method is self-contained, transparent, open source and independently verifiable. Users need no specialist knowledge and it is intended, primarily, for the environmental science community to improve the understanding of aviation's impact upon the environment and to assess the likely efficacy of mitigation strategies. It is also suitable for use in economic analyses, studies to support policy-making and for teaching aircraft performance.

\subsection{THE METHOD}

The complete method, together with the underpinning arguments, is described in detail in Poll and Schumann ${ }^{(2)}$. Therefore, only a brief summary covering the relevant parameters and the key governing relations is presented here.

During cruise, the fuel consumption per unit distance travelled through the air is

$$
\frac{d m_{f}}{d S}=-\frac{d m}{d S}=\frac{m g}{\left(\eta_{o} L / D\right) L C V}
$$

where $m_{f}$ is the instantaneous fuel mass, $m$ is the instantaneous total mass of the aircraft, $S$ is the distance travelled through the air, $L$ is the lift, $D$ is the drag, $g$ is the acceleration due to gravity, $L C V$ is the lower calorific value of the fuel and $\eta_{o}$ is the overall propulsion efficiency of the engines, defined as

$$
\eta_{o}=\frac{F_{n} V_{\infty}}{\dot{m}_{f} L C V}=\frac{D V_{\infty}}{\dot{m}_{f} L C V},
$$

where $F_{n}$ is the total net thrust and $V_{\infty}$ is the true airspeed. Therefore, the aircraft characteristic that governs fuel efficiency is $\left(\eta_{o} L / D\right)$. The application of dimensional analysis shows that $\left(\eta_{o} L / D\right)$ depends upon the atmospheric variation of temperature with pressure, the flight Mach number, $M_{\infty}$, and an aircraft Reynolds number, $R^{a c}$, which is defined as

$$
R^{a c}=\frac{l \rho_{\infty} V_{\infty}}{\mu_{\infty}}=S_{r e f}^{1 / 2}\left(\frac{\rho_{\infty} a_{\infty}}{\mu_{\infty}}\right) M_{\infty}=S_{r e f}^{1 / 2}\left(\frac{\gamma p_{\infty}}{\mu_{\infty} a_{\infty}}\right) M_{\infty} .
$$


Here, air is taken to be an ideal gas, $l$ is a 'typical' aircraft reference length, taken to be the square root of the reference wing area, $S_{r e f}, p_{\infty}$ is the atmospheric static pressure, $\rho_{\infty}$ is the density, $a_{\infty}$ is the local speed of sound, $\mu_{\infty}$ is the dynamic viscosity and $\gamma$ is the ratio of specific heats.

As described in Poll and Schumann ${ }^{(2)}$, for flight at Mach numbers in excess of 0.5 and values of lift coefficient, $C_{L}$, in the cruise range, i.e. 0.4 to 0.7 , the drag polar may be approximated by a relation of the form

$$
C d \approx C d_{0}+\left(\frac{1}{\pi \cdot A R \cdot e_{L S}}\right) C_{L}^{2}+C d_{w},
$$

where $C_{L}$ is defined as

$$
C_{L}=\frac{m g}{(\gamma / 2) p_{\infty} M_{\infty}^{2} S_{r e f}}
$$

$C d_{0}$ is the zero-lift, profile drag coefficient ${ }^{1}, e_{L S}$ is the 'low-speed' Oswald efficiency factor, $A R$ is the wing aspect ratio, defined as

$$
A R=\frac{s^{2}}{S_{r e f}}
$$

where $s$ is the wingspan and $C d_{w}$ is the 'wave drag' coefficient.

The standard approximation is that $C d_{0}$ is directly proportional to the aircraft's mean skin-friction coefficient, $C_{F}^{a c}$, and, for $M_{\infty}$ greater than $0.5, C d_{0}$ depends upon Reynolds number but not Mach number; see e.g. Shevell ${ }^{(4)}$ (chapter 12). This is because, as $M_{\infty}$ increases beyond 0.5 , a near balance is struck between the increase in form drag due to Mach number-induced changes to the surface pressure distribution and the reduction in skin friction due to the surface temperature rise. As Poll and Schumann ${ }^{(2)}$ show, the relationship between skin friction and Reynolds number, at a Mach number of 0.5, can be approximated by a power law, i.e.

$$
\left(C_{F}^{F P}\right)_{M=0.5}=C_{F}^{a c} \approx \frac{a}{\left(R^{a c}\right)^{b}}
$$

where $a$ and $b$ are constants having values of 0.0269 and 0.14 , respectively.

The Oswald factor captures all the lift-dependent drag effects, of which vortex drag on the wings is the primary source. However, the tailplane and the fuselage also generate vortex drag. In addition, there are non-vortex, lift-dependent drag contributions arising because a change in lift alters the pressure distributions over the various components, which, in turn, alters their profile drag. Therefore, the Oswald factor is a complex parameter and, as shown by Shevell ${ }^{(4)}$, it is primarily a function of aircraft geometry and $C d_{0}$. It may also be represented approximately, but nevertheless accurately, by a power law, see Poll and Schumann ${ }^{(2)}$, i.e.

$$
e \approx \frac{E}{\left(C_{F}^{a c}\right)^{\tau}} \quad \text { where } \quad \tau=-\left(\frac{C_{F}^{a c}}{e}\right) \frac{d e}{d C_{F}^{a c}},
$$

${ }^{1}$ Strictly speaking, $C d_{0}$ is not necessarily the true drag coefficient when the lift force is zero but rather a quantity that is derived by fitting Equation (4) to the actual drag polar over the limited $C_{L}$ range. 
where the coefficients $E$ and $\tau$ depend only upon the aircraft geometry and a representative value of $C d_{0}$.

Similarly, the 'wave drag' coefficient, $C d_{w}$, is a complex quantity that captures all the drag resulting from compressibility, the development of regions of supersonic flow at the wing surface and, eventually, the formation of shockwaves. In general, it depends upon Mach number, lift coefficient and, to a lesser extent, Reynolds number. A detailed description of wave drag can be found in Shevell ${ }^{(4)}$.

As shown in Ref. 1, $\left(\eta_{o} L / D\right)$ exhibits an absolute maximum, or optimum, value at a particular combination of Mach number and lift coefficient, i.e. $M_{o}$ and $\left(C_{L}\right)_{o}$. In addition, as demonstrated by Poll and Schumann ${ }^{(2)}$, at the optimum condition, the values of $\left(\eta_{o} L / D\right)_{0},\left(C_{L}\right)_{o}$, $(L / D)_{\mathrm{o}}$ and $M_{o}$ are related to the skin friction coefficient by a series of parameters that are constant, non-dimensional and characteristic of the aircraft. These parameters are given by

$$
\begin{aligned}
& \psi_{1}=\left(\eta_{o} L / D\right)_{o}^{R}\left(C_{F}^{a c}\right)^{\left(\frac{1+\tau}{2}\right)}, \\
& \psi_{2}=\left(C_{L}\right)_{o}^{R}\left(\frac{1}{C_{F}^{a c}}\right)^{\left(\frac{1-\tau}{2}\right)}, \\
& \psi_{3}=\left(\frac{L}{D}\right)_{o}^{R}\left(C_{F}^{a c}\right)^{\left(\frac{1+\tau}{2}\right)}
\end{aligned}
$$

and

$$
\psi_{4}=M_{o}^{R}
$$

Here, the superscript $R$ indicates that the relationship between the variables is that for the situation in which the Reynolds number and, hence, $C_{F}^{a c}$ are constant ${ }^{2}$, whilst the subscript $o$ indicates that the relationships apply at the conditions for which $\left(\eta_{o} L / D\right)$ has its optimum value. Since the analysis will be extended to include the variation of $(L / D)$ with $C_{L}$ and $M_{\infty}$ in a later section, the coefficient $\psi_{3}$ has been included here for completeness.

From Equation (8), $\tau$ is given by

$$
\tau=-\left(\frac{C_{F}^{a c}}{e_{o}}\right)\left(\frac{d e_{o}}{d C_{F}^{a c}}\right),
$$

whilst the last two coefficients are defined as

$$
\psi_{5}=\left(\frac{S_{r e f}^{1 / 2} \psi_{4} \gamma p_{T P}}{\mu_{T P} a_{T P}}\right)_{I S A}
$$

and

$$
\psi_{6}=\left(\frac{M T O M \cdot g}{(\gamma / 2) p_{T P} \psi_{4}^{2} S_{r e f}}\right)_{I S A}
$$

${ }^{2}$ For an aircraft of fixed weight, when $C_{L}$ varies at fixed Mach number, the Reynolds number changes. Therefore, in general, $C_{F}$ and, hence, $C d_{0}$ vary with $C_{L}$. However, if the Reynolds number is constant, $\mathrm{d} C d_{0} / \mathrm{d} C_{L}$ is equal to zero. 
The analysis was extended to include Reynolds number variation in Ref. 2. In this general case, dimensional analysis shows that, in a given atmosphere, the optimum condition is uniquely determined by a Mach number and Reynolds number pair, $M_{o}$ and $R_{o}{ }^{a c}$, where both $M_{o}$ and $\left(C_{L}\right)_{o}$ depend upon $R^{a c}$. The relations governing these quantities are

$$
R_{o}^{a c} \approx G_{2} \psi_{5}\left(\frac{1}{\psi_{7}}\left(\frac{m}{M T O M}\right)\right)^{\iota_{0} \kappa_{o}}
$$

and

$$
M_{o} \approx(1+\varepsilon) \psi_{4}
$$

where the constants $\iota_{\mathrm{o}}, \kappa_{\mathrm{o}}$ and $\varepsilon$ and the function $G_{2}$ are defined and described in Appendix A, MTOM is the aircraft's maximum take-off mass and $\psi_{7}$ is defined as

$$
\psi_{7}=\frac{\psi_{2}}{\psi_{6}}\left(\frac{a}{\psi_{5}^{b}}\right)^{\left(\frac{1-\tau}{2}\right)}
$$

Hence,

$$
\begin{aligned}
& \left(C_{F}^{a c}\right)_{o} \approx \frac{a}{\left(R_{o}^{a c}\right)^{b}}=G_{3}\left(\frac{a}{\psi_{5}^{b}}\right)\left(\psi_{7}\left(\frac{\text { MTOM }}{m}\right)\right)^{b \iota_{o} \kappa_{o}} \\
& \left(C_{L}\right)_{o} \approx G_{4} \psi_{2}\left(\left(\frac{a}{\psi_{5}^{b}}\right)\left(\psi_{7}\left(\frac{M T O M}{m}\right)\right)^{b \iota_{o} \kappa_{o}}\right)^{\left(\frac{1-\tau}{2}\right)}
\end{aligned}
$$

and

$$
\left(\eta_{o} L / D\right)_{o} \approx G_{5} \psi_{1}\left(\left(\frac{\psi_{5}^{b}}{a}\right)\left(\frac{1}{\psi_{7}}\left(\frac{m}{M T O M}\right)\right)^{b \iota_{o} \kappa_{o}}\right)^{\left(\frac{1+\tau}{2}\right)}
$$

The variation of $\left(\eta_{o} L / D\right)$ with lift coefficient and Mach number is then given by

$$
\frac{\left(\eta_{o} L / D\right)}{\left(\eta_{o} L / D\right)_{o}} \approx f_{3}\left(\frac{\left(C_{L}\right)_{o}}{C_{L}}\right)^{i b\left(\frac{1+\tau}{2}\right)}\left(1+\frac{A}{2}\left(\left(f_{4}\left(\frac{C_{L}}{\left(C_{L}\right)_{o}}\right)^{v}\right)-1\right)^{2}+\frac{B}{6}\left(\left(f_{4}\left(\frac{C_{L}}{\left(C_{L}\right)_{o}}\right)^{v}\right)-1\right)^{3}\right)
$$

where the functions $G_{3}$ to $G_{5}, A, B, f_{3}, f_{4}, \iota$ and $v$ are also given in Appendix A.

Therefore, to determine $\left(\eta_{o} L / D\right)_{\mathrm{o}},\left(C_{L}\right)_{o}$ and $M_{o}$ for a given aircraft, in addition to the quantities listed in Appendix A, values must be assigned to five non-dimensional, $\psi$ coefficients and to $\tau$.

\subsection{SOURCES OF DATA}

The number of sources of whole aircraft information available in the public domain is limited.

The first, and most reliable, is the aircraft Type Certificate Data Sheet (TCDS), e.g. Ref. 5. This 
is issued by the regulating authority and lists, amongst other things, the maximum operational Mach number, $M_{M O}$, the maximum permitted flight level, $(F L)_{M O}$ and the maximum permitted values of take-off mass, $M T O M$, landing mass, $M L M$, and zero-fuel mass, $M Z F M$. The second source is the series of Airport Planning Reports ( $A P R s)$ produced by the manufacturers, e.g. Ref. 6. These contain detailed mass breakdowns and external geometry information, together with payload range diagrams and some take-off and landing performance data. However, unlike the TCDS information, the accuracy of $A P R$ data is not guaranteed and there may be inconsistencies, or even errors, in these documents. Thirdly, aircraft external geometry and some performance data, e.g. long-range cruise Mach numbers, can be found in commercial reference sources such as Jane's ${ }^{(7)}$. Engine characteristics and some performance data are also available from Jane's ${ }^{(8)}$ and regulator databases, e.g. $I C A O^{(9)}$. Finally, a limited amount of aerodynamic data appears in standard aircraft design texts, e.g. Jenkinson et al. ${ }^{(10)}$ and Torenbeek ${ }^{(11,12)}$, with a particularly useful collection being given in Obert ${ }^{(13)}$. Again, the accuracy of these sources is not guaranteed, and cross-checking of data is always advisable. In this study, 53 aircraft have been considered, and these are listed in Table 1.

\subsection{THE EVALUATION OF THE ZERO-LIFT, PROFILE DRAG COEFFICIENT}

In standard texts on conceptual design, e.g. Shevell ${ }^{(4)}$, the low-speed, zero-lift drag coefficient is usually determined by first estimating the profile drag of the wing, the fuselage, the empennage and the engine nacelles (outer surface only) in isolation.

Component profile drag is separated into two elements, surface shear, or 'skin friction', and pressure, or 'form', drag. The traditional process involves multiplying the component's exposed, or 'wetted', surface area by the mean skin friction occurring on a flat plate of the same area, at zero incidence to the flight direction and with the same streamwise Reynolds number. A 'form factor' multiplier is then applied to account for the pressure drag and the element of surface shear, over and above the flat-plate value, caused by the component's thickness distribution, its curvature and the effect of the pressure distribution on the boundary layer development. The component drags are then summed and corrections are applied to allow for the interference effects between components and secondary effects, such as excrescences associated with system installation, gaps and other surface imperfections. This process is captured by the expression

$$
\frac{C d_{0}}{\left(C_{F}^{a c}\right)_{M=0.5}}=F_{o} \sum_{0}^{i}\left(F_{F} F_{I} F_{S}\left(\frac{C_{F}}{\left(C_{F}\right)_{a c}}\right)\left(\frac{S_{w e t}}{S_{r e f}}\right)\right)_{i}=\psi_{0},
$$

where $F_{F}$ is the component form factor, $F_{I}$ is the interference factor, $F_{S}$ is the secondary drag factor, $C_{F}$ is the flat-plate skin-friction coefficient for the component, $S_{\text {wet }}$ is the component wetted area and $F_{o}$ is an overall factor intended to capture any other effects. The ratio of the component $C_{F}$ to $\left(C_{F}\right)_{a c}$ is a function of the ratio of the component Reynolds number, $R_{i}$, to the aircraft characteristic Reynolds number, $R^{a c}$, only and this depends upon the ratio of the component streamwise characteristic length, $l$, to the aircraft characteristic length $\left(=S_{r e f}^{1 / 2}\right)$. Approximate values for $F_{F}, F_{S}$ and $F_{I}$ are given, in some form, in most aircraft concept design texts. For consistency, it is important that the factors are clearly defined and, ideally, they 
Table 1

Basic information for a range of turbofan powered civil transport aircraft

\section{Weight}

\begin{tabular}{|c|c|c|c|c|c|c|c|c|c|c|c|}
\hline ICAO & Manufacturer & Type & Engine & BPR(nom) & $\begin{array}{l}\text { Weight } \\
\text { variant }\end{array}$ & MTOM(kg) & $S_{r e f}\left(\mathbf{m}^{2}\right)$ & $s(\mathbf{m})$ & $\boldsymbol{\Lambda}_{\mathrm{w}(\mathrm{deg})}$ & $\mathbf{M}_{\mathbf{M O}}$ & FL $_{M O}$ \\
\hline A30B & Airbus & A300B4-200 & GE CF6-50C & 4.3 & 6 & 165,000 & 260.0 & 44.83 & 28 & 0.82 & 390 \\
\hline A306 & Airbus & A300B4-600R & GE CF6-80C2A1 & 5.1 & 0 & 170,500 & 260.0 & 44.84 & 28 & 0.82 & 410 \\
\hline A310 & Airbus & A310-200 & GE CF6-80C2A2 & 5.1 & 8 & 138,600 & 219.0 & 43.89 & 28 & 0.84 & 410 \\
\hline A313 & Airbus & A310-300 & PW JT9D-7R4 & 5.0 & 0 & 150,000 & 219.0 & 43.89 & 28 & 0.84 & 410 \\
\hline A318 & Airbus & A318-100 & CFM56-5B9_P & 5.9 & 5 & 68,000 & 122.4 & 34.10 & 25 & 0.82 & 410 \\
\hline A319 & Airbus & A319-100 & CFM56-5A5 & 6.0 & 6 & 73,500 & 122.4 & 34.10 & 25 & 0.82 & 410 \\
\hline A320 & Airbus & A320-200 & CFM56-5B4_P & 5.9 & 0 & 73,500 & 122.4 & 34.10 & 25 & 0.82 & 410 \\
\hline A321 & Airbus & A321-100 & CFM56-5B1 & 5.7 & 8 & 89,000 & 122.4 & 34.15 & 25 & 0.82 & 391 \\
\hline A332 & Airbus & A330-200 & RR Trent772 & 5.0 & 52 & 233,000 & 361.6 & 60.30 & 30 & 0.86 & 410 \\
\hline A333 & Airbus & A330-300 & PW4164 & 5.2 & 52 & 233,000 & 361.6 & 60.30 & 30 & 0.86 & 410 \\
\hline A342 & Airbus & A340-200 & CFM56-5C4 & 6.6 & 1 & 257,000 & 361.6 & 60.30 & 30 & 0.86 & 415 \\
\hline A343 & Airbus & A340-300 & CFM56-5C4 & 6.6 & 1 & 257,000 & 361.6 & 60.30 & 30 & 0.86 & 415 \\
\hline A345 & Airbus & A340-500 & RR Trent553-61 & 7.5 & 1 & 372,000 & 437.3 & 63.45 & 31 & 0.86 & 415 \\
\hline A346 & Airbus & A340-600 & RR Trent556-61 & 7.5 & 1 & 368,000 & 437.3 & 63.45 & 31 & 0.86 & 415 \\
\hline A359 & Airbus & A350-900 & RR TrentXWB-84 & 9.0 & 9 & 275,000 & 445.0 & 64.75 & 32 & 0.89 & 431 \\
\hline A388 & Airbus & A380-800 & EA GP7270 & 8.7 & 2 & 569,000 & 845.0 & 79.80 & 30 & 0.89 & 431 \\
\hline B712 & Boeing & B717-200 & RR BR700-715C1-30 & 4.6 & HGW & 54,884 & 92.8 & 28.40 & 25 & 0.82 & 371 \\
\hline B732 & Boeing & B737-200 & PW JT8D-15 & 1.0 & 5 & 52,390 & 99.0 & 28.35 & 25 & 0.84 & 370 \\
\hline B733 & Boeing & B737-300 & CFM56-3-B1 & 5.1 & 3 & 61,236 & 102.0 & 28.90 & 25 & 0.82 & 390 \\
\hline B734 & Boeing & B737-400 & CFM56-3C-1 & 5.1 & 6 & 68,039 & 102.5 & 28.90 & 25 & 0.82 & 370 \\
\hline B735 & Boeing & B737-500 & CFM56-3-B1 & 5.1 & 3 & 60,555 & 103.7 & 28.90 & 25 & 0.82 & 370 \\
\hline B736 & Boeing & B737-600 & CFM56-7B22 & 5.3 & 3 & 65,544 & 124.6 & 34.30 & 25 & 0.82 & 410 \\
\hline B737 & Boeing & B737-700 & CFM56-7B24 & 5.2 & 3 & 70,080 & 124.6 & 34.30 & 25 & 0.82 & 410 \\
\hline B738 & Boeing & B737-800 & CFM56-7B26 & 5.1 & 3 & 79,016 & 124.6 & 34.30 & 25 & 0.82 & 410 \\
\hline B739 & Boeing & B737-900ER & CFM56-7B26 & 5.1 & 2 & 85,139 & 124.6 & 34.32 & 25 & 0.82 & 410 \\
\hline B742 & Boeing & B747-200B & RB211-524C2 & 4.5 & 4 & 371,900 & 511.0 & 59.64 & 38 & 0.92 & 450 \\
\hline B743 & Boeing & B $747-300$ & PW JT9D-7R4 & 5.0 & 0 & 377,800 & 511.0 & 59.64 & 39 & 0.90 & 450 \\
\hline
\end{tabular}


Table 1

Continued

\begin{tabular}{|c|c|c|c|c|c|c|c|c|c|c|c|}
\hline ICAO & Manufacturer & Type & Engine & BPR(nom) & $\begin{array}{l}\text { Weight } \\
\text { variant }\end{array}$ & MTOM(kg) & $S_{r e f}\left(\mathbf{m}^{2}\right)$ & $s(\mathbf{m})$ & $\boldsymbol{\Lambda}_{\mathrm{w}(\mathrm{deg})}$ & $\mathbf{M}_{\mathbf{M O}}$ & $\mathbf{F L}_{\mathbf{M O}}$ \\
\hline B744 & Boeing & B747-400 & GE CF6-80C2B1F & 5.1 & 5 & 396,894 & 547.0 & 64.44 & 38 & 0.92 & 450 \\
\hline B748 & Boeing & B747-8F & GEnx-2B67 & 8.0 & 1 & 442,253 & 594.0 & 68.40 & 38 & 0.90 & 421 \\
\hline B752 & Boeing & B757-200 & RR RB211-535C & 4.5 & 4 & 113,400 & 189.0 & 38.06 & 25 & 0.86 & 420 \\
\hline B753 & Boeing & B757-300 & RR RB211-535E4B & 4.1 & 2 & 122,470 & 189.0 & 38.06 & 25 & 0.86 & 430 \\
\hline B762 & Boeing & B767-200ER & PW4056 & 4.7 & 6 & 179,169 & 283.3 & 47.57 & 32 & 0.86 & 430 \\
\hline B763 & Boeing & B767-300 & PW4060 & 4.5 & 2 & 158,758 & 283.3 & 47.57 & 32 & 0.86 & 431 \\
\hline B764 & Boeing & B767-400ER & GE CF6-80C2B8F & 5.1 & 1 & 204,116 & 283.3 & 51.92 & 32 & 0.86 & 450 \\
\hline B77L & Boeing & B777-200LR & GE90-110B1 & 7.3 & 1 & 347,450 & 427.8 & 64.80 & 32 & 0.89 & 431 \\
\hline B772 & Boeing & B777-200 & RR Trent892 & 5.7 & 6 & 286,900 & 427.8 & 60.93 & 32 & 0.89 & 431 \\
\hline B77W & Boeing & B777-300ER & GE90-115B & 7.1 & 1 & 351,530 & 427.8 & 64.80 & 32 & 0.89 & 431 \\
\hline B773 & Boeing & B777-300 & RR Trent892 & 5.7 & 4 & 299,370 & 427.8 & 60.93 & 32 & 0.89 & 431 \\
\hline B788 & Boeing & B787-8 & GEnx-1B64 & 9.0 & 1 & 227,930 & 360.0 & 60.12 & 32 & 0.90 & 431 \\
\hline B789 & Boeing & B787-9 & RR Trent1000-AE3 & 9.2 & 1 & 254,011 & 360.0 & 60.12 & 32 & 0.90 & 431 \\
\hline E135 & Embraer & EMB-135LR & RR AE3007A & 5.2 & 0 & 20,000 & 51.2 & 20.04 & 23 & 0.78 & 370 \\
\hline E145 & Embraer & EMB-145LR & RR AE3007A1 & 4.8 & 0 & 22,000 & 51.2 & 20.04 & 23 & 0.78 & 370 \\
\hline E170 & Embraer & EMB-170LR & GE CF34-8E5A1 & 5.1 & 0 & 37,200 & 72.7 & 25.30 & 23 & 0.82 & 410 \\
\hline E195 & Embraer & EMB-195STD & GE CF34-10E5A1 & 5.1 & 0 & 48,790 & 92.5 & 27.73 & 23 & 0.82 & 410 \\
\hline MD82 & Boeing & MD-82 & PW JT8D-217A & 1.7 & 0 & 67,812 & 112.3 & 32.85 & 23 & 0.84 & 370 \\
\hline MD83 & Boeing & MD-83 & PW JT8D-219 & 1.7 & 0 & 72,575 & 112.3 & 32.85 & 23 & 0.84 & 370 \\
\hline GLF5 & Gulfstream & G-550 & RR BR700-710C4-11 & 4.1 & 0 & 41,277 & 105.6 & 28.50 & 25 & 0.89 & 510 \\
\hline CRJ9 & Bombardier & CRJ-9 & GE CF34-8C5 & 5.1 & 0 & 38,329 & 76.2 & 23.25 & 26 & 0.85 & 410 \\
\hline DC93 & Boeing & DC-9-32 & PW JT8D-7series & 1.1 & 0 & 48,988 & 93.0 & 28.44 & 24 & 0.84 & 370 \\
\hline $\mathrm{RJ} 1 \mathrm{H}$ & BAESystems & RJ-100 & LF507-1F-1H & 5.1 & 0 & 44,225 & 77.3 & 26.34 & 15 & 0.73 & 350 \\
\hline B722 & Boeing & $727-200$ & PW JT8D-15 & 1.0 & 0 & 83,820 & 157.9 & 32.92 & 32 & 0.90 & 420 \\
\hline A20N & Airbus & A320-NEO & PW1127G & 12.2 & 54 & 79,000 & 122.4 & 35.10 & 25 & 0.82 & 410 \\
\hline $\mathrm{A} 21 \mathrm{~N}$ & Airbus & A321-NEO & CFM LEAP-1A32 & 10.5 & 52 & 93,500 & 122.4 & 35.27 & 25 & 0.82 & 391 \\
\hline
\end{tabular}


should all come from the same source. Unfortunately, there appears to be no single reference that provides all the elements and the present calculation scheme, summarised in Appendix $\mathrm{B}$, is based upon relations proposed by Jenkinson et al. ${ }^{(10)}$ and Torenbeek ${ }^{(11,12)}$. The term $F_{o}$ is not specified 'a priori'; rather, it is used for the final calibration of the $C d_{o}$ estimates against wind-tunnel or flight-test data. As can be seen from Equation (23), $\psi_{0}$ depends only upon the aircraft geometry.

The estimation is completed by the specification of a relation linking the skin-friction coefficient and the Reynolds number, i.e. Equation (7). It should be noted that the use of this relation implies that the boundary layers on the aircraft are turbulent everywhere. For large aircraft, this is almost always the case; see Poll ${ }^{(14)}$. However, for smaller aircraft such as business jets, there may be significant areas of laminar flow on the wings and the empennage. In addition, in recent times, attempts have been made to establish small areas of laminar flow on the engine nacelles and empennage of the Boeing 787 and on the winglets of the Boeing 737 Max series. Therefore, if an aircraft is known to have significant regions of laminar flow during cruise, a correction should be applied on a component-by-component basis.

Ideally, the estimation method should be calibrated against flight-test data, but such information rarely appears in the public domain. However, Obert ${ }^{(13)}$ (figure 40.17) gives a graph of 'high-speed' subsonic $C d_{o}$, derived from trimmed, flight-test drag polars of the form given in Equation (4), versus aircraft total wetted area for 26 civil transport aircraft. Twelve of these aircraft are amongst those listed in Table 1 and the maximum scatter relative to the mean line is about $\pm 10 \%$. To generate comparative values for those 39 aircraft for which Obert does not provide a specific data point, functions linking Obert's $C d_{o}$ values to the aircraft's total wetted area were produced by simple curve fitting of the mean line. Each aircraft's total wetted area was then estimated using the relations given in Appendix B and the curve fit was used to generate an 'Obert' value for $C d_{o}$. These interpolated values are subject to the same uncertainty as the data upon which they are based, i.e. $\pm 10 \%$.

Unfortunately, Obert specifies neither the test Mach number nor the Reynolds number for his data. However, the test conditions are described as 'average CG (centre of gravity) position', 'Mach numbers greater than 0.4 ' and no 'compressibility effects'. This last statement is taken to mean that the Mach numbers are below those at which wave drag is significant, i.e. $C d_{w}$ is effectively zero. Therefore, when producing the present $C d_{0}$ estimates from Equations (7) and (23), in the absence of specific information to the contrary, cruise Mach numbers are taken to be $65 \%$ of the maximum permitted operational value, $M_{M O}$, giving values between 0.5 and 0.6 , whilst the cruise altitude is set to $85 \%$ of the maximum permitted operating height, giving values in the range of 30,000 to $40,000 \mathrm{ft}$. International Standard Atmosphere conditions $^{(15)}$ are assumed. Maximum permitted Mach numbers and altitudes are both listed in the $T C D S$. The maximum deviation in $C d_{o}$ resulting from any mismatch between the assumed and actual Reynolds number is estimated to be less than $3 \%$.

Figure 1 shows a comparison between the Obert data and the estimates for $C d_{o}$ for all the aircraft listed in Table 1 with the overall factor, $F_{o}$, set to 0.98 . All the $C d_{o}$ values lie within the scatter exhibited by the original Obert data, with the maximum deviation for any individual point being about $10 \%$. Given the intrinsic uncertainties in the approach, these differences are not considered to be significant. The estimated values of $\Psi_{0}$ for the 53 aircraft types are given in Table 2. 
Table 2

\section{Estimates of the characteristic parameters of a range of turbofan-powered} civil transport aircraft

\begin{tabular}{|c|c|c|c|c|c|c|c|c|c|c|}
\hline AO & $\eta_{2}$ & $\psi_{0}$ & $\tau$ & $\psi_{1}$ & $\psi_{2}$ & $\psi_{3}$ & $\psi_{4}$ & $\psi_{5}$ & $\psi_{6}$ & $\eta_{1}$ \\
\hline 30B & , & 2 & 20 & 46 & 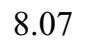 & 52 & 53 & 07 & 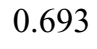 & . \\
\hline A306 & & & & & & & 753 & & & \\
\hline 310 & 539 & & & 62 & .12 & 20 & .772 & & & 360 \\
\hline 313 & & & & & 15 & & 772 & & & \\
\hline & & .532 & 57 & & .02 & 33 & .753 & 07 & .607 & 346 \\
\hline 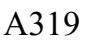 & & & & & .06 & & & & & \\
\hline & & 6 & & & .05 & & 53 & & & 351 \\
\hline & 0 & & & & 8.13 & & 53 & 07 & & \\
\hline & & & & & 17 & & & & & \\
\hline & 6 & 6 & & 94 & 8.18 & 12 & 0.786 & $3 \mathrm{E}+08$ & & 360 \\
\hline & & & & & 8.23 & & & & & \\
\hline & 2 & .127 & & 86 & 8.24 & 78 & 786 & 08 & & 362 \\
\hline & & & & & 8.25 & & 796 & & & \\
\hline & & 5 & & 08 & 8.26 & & 796 & & & 396 \\
\hline & & .477 & & 44 & 8.09 & & .820 & 08 & & 423 \\
\hline & & & & & 08 & & 20 & & & \\
\hline & 0 & 653 & & 121 & 8.00 & 62 & 0.724 & 07 & & 315 \\
\hline & & & & & 7.99 & & 685 & & & 277 \\
\hline & & 5 & & 28 & 8.03 & & 0.729 & & & 310 \\
\hline & & & & & & & & & & \\
\hline & & & & & 8.01 & & 729 & & & 302 \\
\hline & & & & & 8.00 & & 58 & & & 323 \\
\hline & & 0 & & & 8.03 & & 758 & & & 331 \\
\hline & & .943 & & & 8.08 & & 758 & & & 334 \\
\hline & & 3 & & 45 & 8.12 & 00 & 758 & 07 & & 338 \\
\hline & & & & & 7.73 & & & & & 319 \\
\hline & & & & & & & & & & \\
\hline & & & & & 7.84 & & & $7 \mathrm{E}+08$ & & 344 \\
\hline & & & & & 7.93 & & & $3 \mathrm{E}+08$ & & 387 \\
\hline & & & & & 8.05 & & 0.772 & & & 365 \\
\hline & & & & & 8.11 & & 0.772 & $2 \mathrm{E}+07$ & & 351 \\
\hline & 0 & 6.5 & 16 & 99 & 7.93 & 06 & 0.772 & $E+07$ & 57 & 379 \\
\hline & & & & & 7.93 & & 0.772 & & & 0.382 \\
\hline & 0 & 7.173 & 6 & 82 & 8.12 & 66 & 0.772 & $E+07$ & 48 & 370 \\
\hline & & 6.636 & & 0.217 & 8.23 & 0.620 & 0.811 & $1.27 \mathrm{E}+08$ & & .387 \\
\hline & & & & & & & & $7 \mathrm{E}+08$ & & \\
\hline $77 \mathrm{~W}$ & & 6.991 & & 0.219 & 8.25 & & 0.811 & $1.27 \mathrm{E}+08$ & & 410 \\
\hline & & & & & 8.15 & & & $1.27 \mathrm{E}+08$ & & .388 \\
\hline & & 6.621 & & 0.238 & 8.07 & 0.610 & 0.815 & $1.17 \mathrm{E}+08$ & 0.589 & .424 \\
\hline & & & & & 8.13 & & & $1.17 \mathrm{E}+08$ & & .426 \\
\hline & & 7.861 & & 0.109 & 7.75 & 0.493 & 0.704 & $3.81 \mathrm{E}+07$ & 0.487 & 0.266 \\
\hline E145 & 0.550 & 8.215 & 0.152 & 0.115 & 7.80 & 0.475 & 0.704 & $3.81 \mathrm{E}+07$ & 0.536 & 0.294 \\
\hline
\end{tabular}




\section{Table 2}

\section{Continued}

$\begin{array}{lcccccccccc}\text { ICAO } & \boldsymbol{\eta}_{\mathbf{2}} & \boldsymbol{\psi}_{\mathbf{0}} & \boldsymbol{\tau} & \boldsymbol{\psi}_{\mathbf{1}} & \boldsymbol{\psi}_{\mathbf{2}} & \boldsymbol{\psi}_{\mathbf{3}} & \boldsymbol{\psi}_{\mathbf{4}} & \boldsymbol{\psi}_{\mathbf{5}} & \boldsymbol{\psi}_{\mathbf{6}} & \boldsymbol{\eta}_{\mathbf{1}} \\ \text { E170 } & 0.538 & 8.210 & 0.162 & 0.126 & 7.94 & 0.483 & 0.733 & 4.72 \mathrm{E}+07 & 0.589 & 0.308 \\ \text { E195 } & 0.540 & 8.165 & 0.152 & 0.137 & 8.00 & 0.490 & 0.758 & 5.50 \mathrm{E}+07 & 0.569 & 0.324 \\ \text { MD82 } & 0.646 & 8.323 & 0.169 & 0.116 & 8.10 & 0.487 & 0.753 & 6.03 \mathrm{E}+07 & 0.660 & 0.287 \\ \text { MD83 } & 0.646 & 8.323 & 0.167 & 0.122 & 8.13 & 0.489 & 0.753 & 6.03 \mathrm{E}+07 & 0.706 & 0.299 \\ \text { GLF5 } & 0.572 & 6.571 & 0.122 & 0.188 & 7.71 & 0.587 & 0.772 & 5.99 \mathrm{E}+07 & 0.406 & 0.371 \\ \text { CRJ9 } & 0.538 & 7.322 & 0.123 & 0.147 & 7.75 & 0.529 & 0.753 & 4.96 \mathrm{E}+07 & 0.550 & 0.323 \\ \text { DC93 } & 0.667 & 8.114 & 0.155 & 0.100 & 7.98 & 0.492 & 0.733 & 5.34 \mathrm{E}+07 & 0.606 & 0.250 \\ \text { RJ1H } & 0.539 & 9.286 & 0.172 & 0.094 & 8.20 & 0.441 & 0.676 & 4.49 \mathrm{E}+07 & 0.776 & 0.263 \\ \text { B722 } & 0.668 & 7.055 & 0.114 & 0.127 & 7.66 & 0.543 & 0.830 & 7.88 \mathrm{E}+07 & 0.477 & 0.264 \\ \text { A20N } & 0.316 & 7.978 & 0.189 & 0.175 & 8.00 & 0.502 & 0.753 & 6.29 \mathrm{E}+07 & 0.705 & 0.382 \\ \text { A21N } & 0.369 & 8.584 & 0.197 & 0.152 & 8.04 & 0.468 & 0.753 & 6.29 \mathrm{E}+07 & 0.835 & 0.360\end{array}$

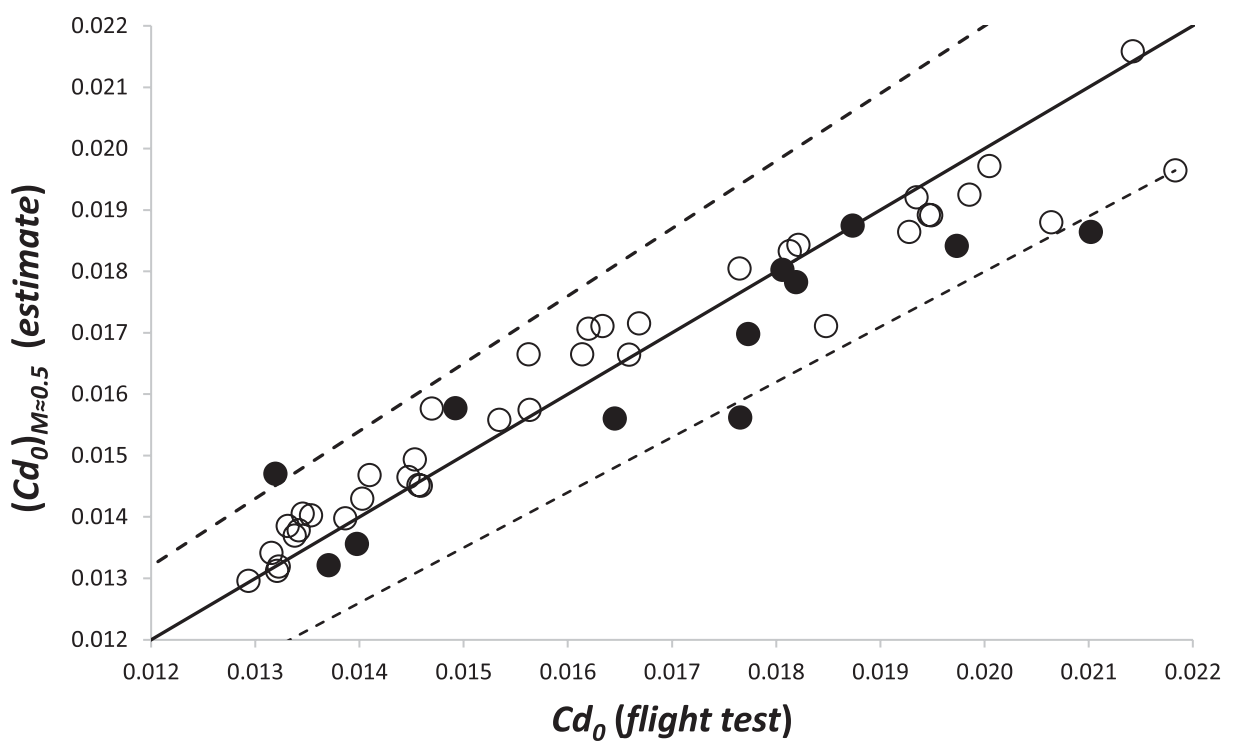

Figure 1. A comparison between the zero-lift drag coefficient $\left(C d_{0}\right)$ estimates from Equations (7) and (23) and the high-speed, flight-test values from Obert ${ }^{(13)}$. Solid symbols are comparisons with an Obert data point. Open symbols indicate a comparison with an estimated 'Obert' value based upon the aircraft total wetted area. The dashed lines indicate $\mathrm{a} \pm 10 \%$ variation.

\subsection{THE ESTIMATION OF THE LIFT-DEPENDENT DRAG COEFFICIENT}

The low-speed, lift-dependent drag coefficient appearing in Equation (4) can be broken down into four elements,

$$
\left(\frac{1}{\pi \cdot A R \cdot e_{L S}}\right)=\left(\frac{1}{\pi \cdot A R}\right)+\left(\frac{\delta_{1}}{\pi \cdot A R}\right)+\left(\frac{\delta_{2}}{\pi \cdot A R}\right)+k_{1}
$$


The first is the incompressible induced, or vortex, drag for an isolated wing having the same aspect ratio as the reference wing and an elliptic spanwise load distribution; see, for example, Shevell $^{(4)}$ (chapter 9). This is the absolute minimum drag that the configuration can have. However, in practice, wing designs never quite achieve an elliptic load distribution and the second term, $\delta_{I}$, captures the additional drag resulting from deviations from the ideal due to the effects of Mach number, taper ratio and sweep, plus those of the spanwise variations in camber and twist. This term can be determined to a high level of accuracy by standard theoretical methods, e.g. ESDU ${ }^{(16)}$ or Computational Fluid Dynamics (CFD). However, despite the dependence upon a great many variables, for a civil transport aircraft its value is found to be very small compared with unity. References (4) and (9) to (13) suggest that typical values of $\delta_{1}$ lie in the range 0.01 to 0.05 , with a reasonable average being about 0.03 .

When a wing is combined with a fuselage, there is mutual interference between the two components. The fuselage generates some lift and the wing experiences both additional downwash and an increase in streamwise velocity, both of which vary across the span. The net result is that the induced drag of the wing is increased by an amount that depends, principally, upon the ratio of the fuselage diameter, $b_{f}$, to the wingspan and this is captured by the coefficient $\delta_{2}$. For a more detailed discussion of this effect see, for example, McLean ${ }^{(17)}$ and Shevell( ${ }^{(4)}$. Using unpublished data, believed to be from the Douglas Aircraft Company, Shevell ${ }^{(4)}$ gives a graph of $\delta_{2}$ versus $b_{f} / \mathrm{s}$ (figure 11.7). This graph suggests that this small effect is well represented by the relation

$$
\delta_{2} \approx 2\left(\frac{b_{f}}{s}\right)^{2}
$$

Finally, $k_{l}$ accounts for additional vortex drag due to any aerodynamic loading on the tailplane and all the non-vortex, lift-dependent drag of the wing, fuselage and tailplane. This factor also depends upon many variables, including the location of the aircraft's centre of gravity. However, it is determined, primarily, by the lift-dependent form drag of the wing. Therefore, whilst, in principle, it can be obtained from theory, or CFD, arguably, this quantity is best obtained from either flight-test data, or from empirical relations derived from flight-test data.

In Ref. 13 (figure 40.36), Obert provides a flight-test dataset that is consistent with, and applicable to, the same flight conditions as the 'high-speed' subsonic $C d_{o}$ values given in his figure 40.17. Obert gives the induced drag factor for 12 of the aircraft listed in Table 1 as a function of aspect ratio and, by taking $\delta_{1}$ to be 0.03 and $\delta_{2}$ to be given by Equation (25), these data can be used to provide estimates for $k_{l}$. Moreover, in cases where derivative aircraft are based upon the same wing design, or one with only minor modifications, e.g. the Airbus A318 to A321 family, the basic values can be attributed, without modification, to more of the aircraft in Table 1.

When no empirical data are available, an estimation relation is needed. Such relations have been proposed, e.g. by Shevell ${ }^{(4)}$ (figure 11.8), and, as noted by Poll and Schumann ${ }^{(2)}$, Shevell's values are well represented by the relation

$$
k_{1} \approx 0.80\left(1-0.53 \cos \left(\Lambda_{w}\right)\right)\left(C d_{o}\right)_{M=0.5}
$$

where $\Lambda_{w}$ is the wing's quarter-chord sweep angle. Shevell does not give any indication of accuracy. However, whilst Obert's results exhibit large type-to-type variation, with $k_{l}$ ranging from 0.004 to 0.012 , a 'least squares' fit suggests that Obert's values follow the general trend proposed by Shevell, but with slightly different values for the coefficients, i.e. 


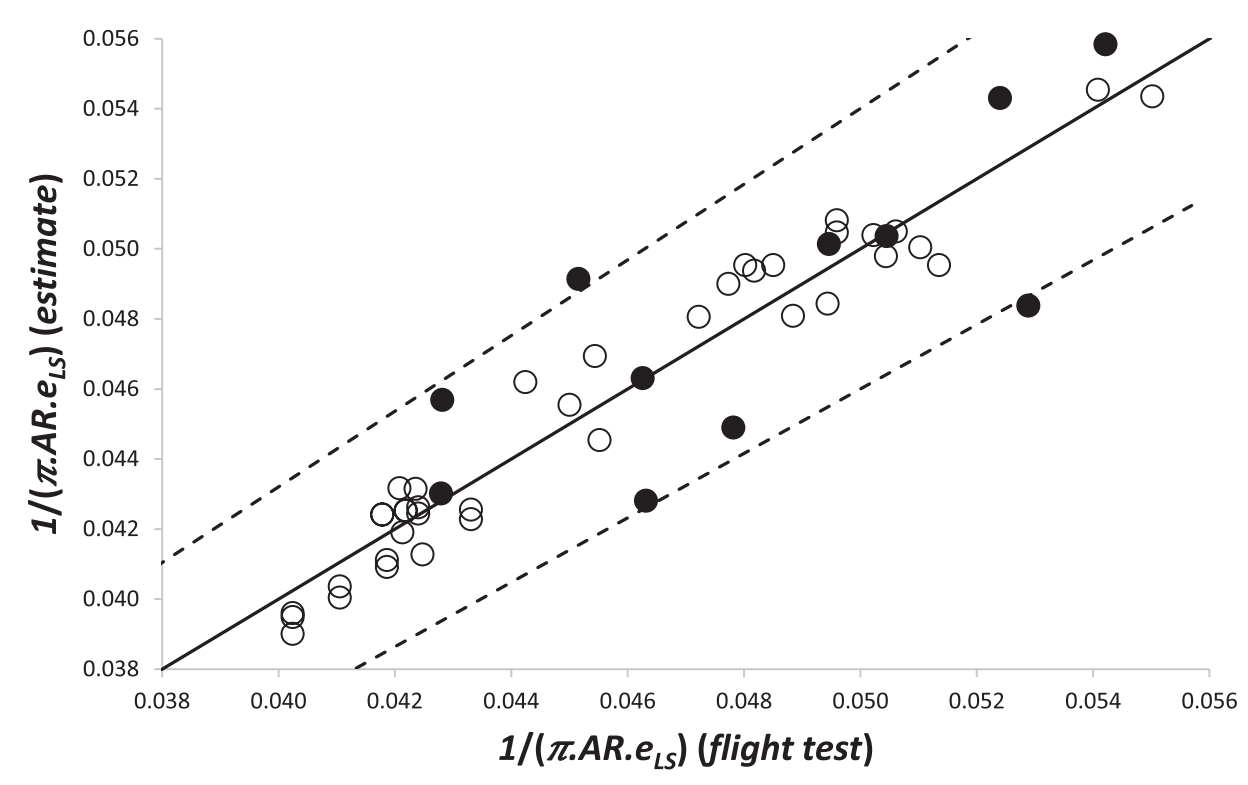

Figure 2. A comparison between the estimated lift-dependent drag coefficient and flight test values from the Obert ${ }^{(13)}$. Test conditions are described as 'average centre of gravity position' and 'Mach numbers greater than 0.4 ' but with no 'compressibility effects'. Solid symbols are comparisons with actual Obert values. Open symbols indicate a comparison with values from Obert's mean line based upon aspect ratio. The dashed lines indicate $\mathrm{a} \pm 8 \%$ variation.

$$
k_{1} \approx 0.83\left(1-0.55 \cos \left(\Lambda_{w}\right)\right)\left(C d_{o}\right)_{M=0.5}
$$

This relation is subject to a maximum spread of about $\pm 50 \%$. However, for the range of sweep angles of practical interest, i.e. $0-40^{\circ}$, Equations (26) and (27) differ by less than $1 \%$. This is taken as confirmation that Equation (26) is valid. The large uncertainty swamps any small variations in $C d_{o}$ due to changes in Reynolds number.

Estimates for the low-speed Oswald factor, $\mathrm{e}_{L S}$, have been obtained from Equation (24), taking $\delta_{1}$ to be $0.03, \delta_{2}$ from Equation (25) and $k_{1}$ from Equation (26). These have then been used to generate the lift-dependent, drag coefficient for each aircraft. A comparison between the present values and either Obert's data points, where possible, or estimates generated from Obert's own mean line through his data is given in Fig. 2. Obert assumes that $k_{l}$ has a constant value of 0.007 , with the sum of $\delta_{1}$ and $\delta_{2}$ being 0.05 . However, since the deviations are less than $\pm 8 \%$, it may be concluded that $e_{L S}$ is not particularly sensitive to the large uncertainties implicit in the estimate of $k_{l}$.

Therefore, there are two ways to approach the problem of estimating the low-speed Oswald factor. The first is to use the values of $k_{l}$ derived from Obert's figure 40.36 for those aircraft to which they apply and to their derivative types. However, the absolute accuracy of the Obert values is not known. The alternative is to use Equation (26) in all cases. However, this equation is known to have a maximum uncertainty of about $\pm 50 \%$. At this stage in the development of the method, Equation (26) has been used for all the aircraft. Further work is required if this uncertainty is to be reduced.

Finally, many modern aircraft have wing tip devices to reduce cruise drag and some designs can be retrofitted to older aircraft. Whilst the detailed characteristics of these devices vary considerably and a full analysis of their aerodynamic effects is a complex task, their overall 
effect is to increase the value of the Oswald factor. According to Torenbeek ${ }^{(12)}$, practical devices increase the Oswald factor by between 5\% and 10\%. Therefore, noting the basic level of uncertainty in the estimation method and in the interests of simplicity, if an aircraft has wing tip devices of any kind, the Oswald factor, based upon the wing without the devices fitted, should be increased by $7.5 \%$.

\subsection{THE ESTIMATION OF THE WAVE-DRAG COEFFICIENT}

As the flight Mach number increases from an initial low value, sonic conditions will eventually be reached at the point on the aerofoil surface where the local static pressure has its lowest value. Usually, this lies close to the leading edge on the wing's upper surface. Further increases in speed result in the formation of a region of supersonic flow that is bounded by the wing surface, a 'sonic interface' ${ }^{3}$ and, if the Mach number is sufficiently high, a terminating shockwave. Once such a 'supersonic zone' is established, when either $M_{\infty}$ or $C_{L}$ is increased, the aircraft drag rises and the terminating shockwave moves rearwards. Whilst this zone is confined to the front portion of the wing, the drag increases are modest, being typically less than 10 drag counts ${ }^{4}$, and the variation is known as 'drag creep'. However, when the terminating shockwave moves onto the rear part of the wing, the rate of drag rise with increasing Mach number, or increasing $C_{L}$, becomes very large and this is usually referred to as 'drag rise'. Since both the creep and rise regimes are linked to the formation and subsequent development of shockwaves, the resulting drag increments are collectively known as 'wave' drag.

Wave drag is a complex phenomenon. However, Shevell ${ }^{(4,18)}$ has developed an approximate method, albeit in fragmented form, for the estimation of wave drag in two-dimensional flow as a function of lift coefficient and the wing thickness-to-chord ratio. No indication of accuracy is provided. However, once again it is highly likely that the method was developed using data from the Douglas Aircraft aerodynamics section. Curve fitting Shevell's graphs and applying streamwise shear to extend the relations to cover the infinite-swept wing ${ }^{5}$ case gives a simple, first order method for the estimation of the element of wave drag, $\delta D_{w}$, acting on a thin streamwise strip of wing with length $c$ and width $\delta y$. Here, $c$ is the streamwise wing chord and $y$ is the spanwise distance measured along the normal to the fuselage centre line. The model suggests that the element of wave drag is a function of Mach number, wing geometry and the element of lift, $\delta L$, also acting on the strip, i.e.

$$
\begin{aligned}
\frac{\delta D_{w}}{(\gamma / 2) p_{\infty} M_{\infty}^{2} c \delta y} \approx & \cos ^{3}\left(\Lambda_{w}\right)\left(0.016\left(\frac{M_{\infty} \cos \left(\Lambda_{w}\right)}{M_{r e f}}-0.75\right)^{2}\right. \\
& \left.+6.5\left(\frac{M_{\infty} \cos \left(\Lambda_{w}\right)}{M_{\text {ref }}}-0.92\right)^{4}\right)
\end{aligned}
$$

\footnotetext{
${ }^{3}$ The surface formed by all the points in the flow field where the local Mach number is unity.

${ }^{4} 1$ drag count is equal to a change of 0.0001 in $C d$.

${ }^{5} \mathrm{An}$ infinite-swept wing is one in which there are no variations in geometry, or flow properties, in the direction parallel to the leading edge.
} 


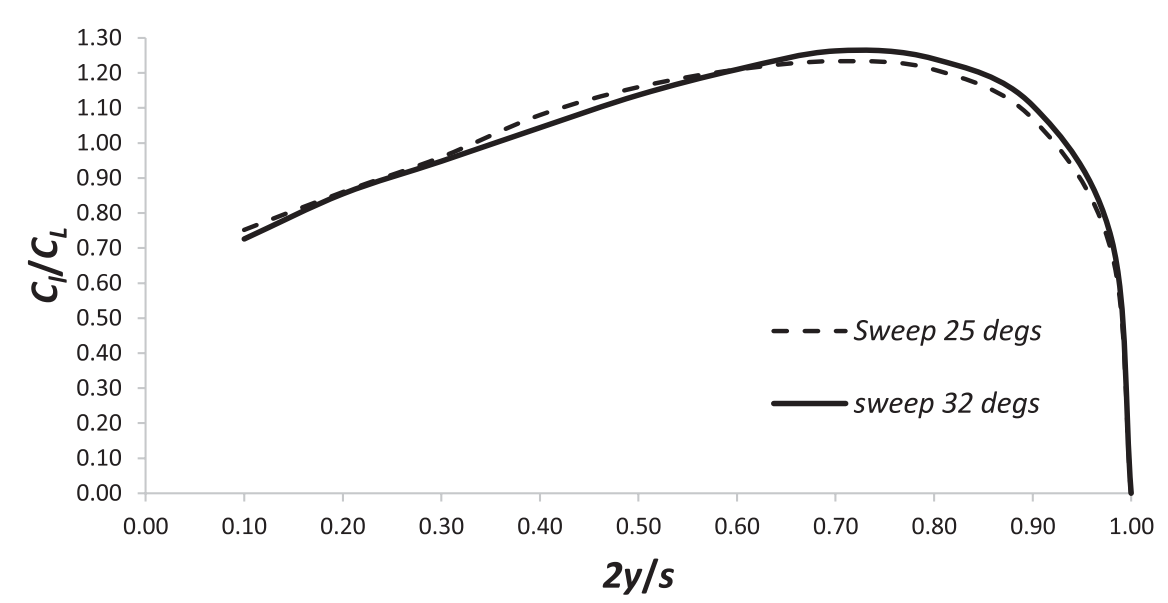

Figure 3. The typical variation of the ratio of the local lift coefficient, $C_{l}$, to the overall lift coefficient, $C_{L}$, with spanwise location for two civil transport aircraft wings having different sweep angles (data from Obert ${ }^{(13)}$, chapter 24).

where

$$
M_{r e f}=0.90-0.10\left(\frac{C_{l}}{\cos ^{2}\left(\Lambda_{w}\right.}\right)-0.96\left(\frac{t / c}{\cos \left(\Lambda_{w}\right)}\right),
$$

$t$ is the maximum wing thickness and the local lift coefficient, $C_{l}$, is given by

$$
C_{l}=\frac{\delta L}{(\gamma / 2) p_{\infty} M_{\infty}^{2} c \delta y}
$$

To estimate the total wave drag for a finite wing, it is necessary to know the spanwise distribution of both $C_{l}$ and the thickness-to-chord ratio. However, modern civil transport wings tend to have similar spanwise variations of chord, $t / c$ and lift; see, for example, Obert ${ }^{(13)}$ (chapter 24). Exploiting this observation, typical normalised lift distributions for sweep angles of $25^{\circ}$ and $32^{\circ}$ have been generated from the wing case studies presented by Obert and these are shown in Fig. 3. The effect of sweep angle is found to be small, but not insignificant.

In addition, on the inner part of the wing, where $(2 y / s)$ is less than about 0.35 , the typical thickness-to-chord ratio distribution is given by

$$
\frac{t}{c} \approx 0.174-0.24\left(\frac{2 y}{s}\right)
$$

whilst on the outer part of the wing $((2 y / s)>0.35), t / c$ is constant with a value of about 0.09 . Combining these approximate variations with Equations (28) to (30) allows an estimate of the distribution of the wave drag across the wing to be made. Finally, integration of the elemental wave drag acting on the strips over the span yields the wing's total wave drag.

Whilst this is undeniably a crude estimate, in practical operations, the wave drag component is always small, being typically less than $10 \%$ of the zero-lift, profile drag component. Consequently, any errors introduced by inaccurate modelling of the wave drag will be small. 


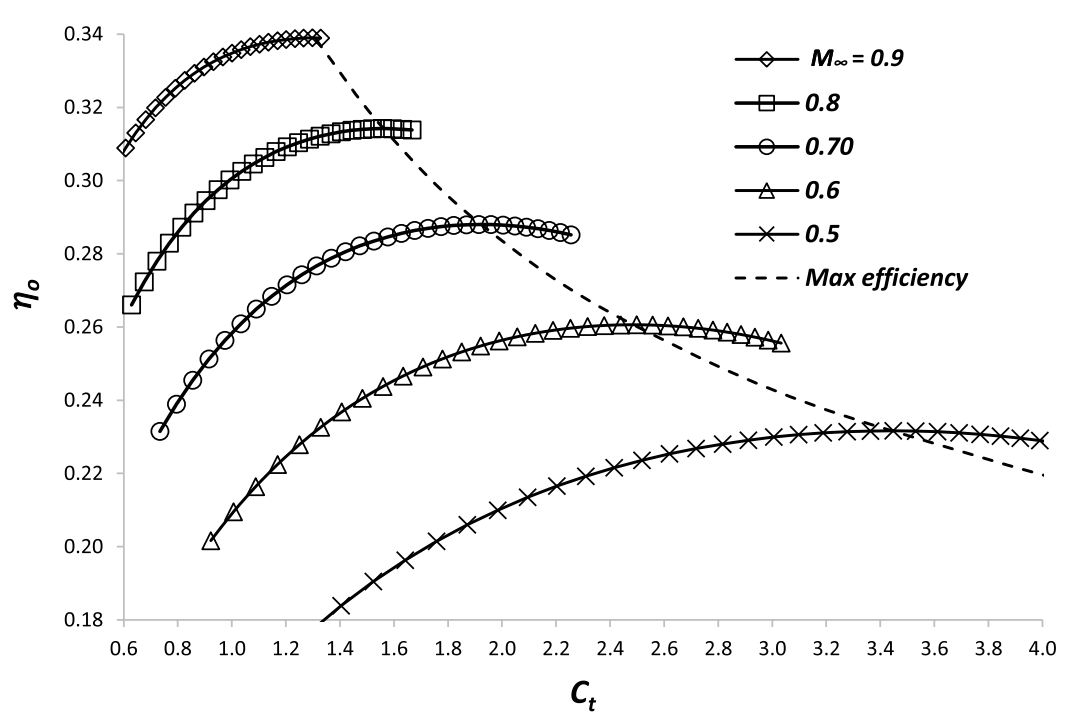

Figure 4. The variation of the engine overall efficiency, $\eta_{o}$, with thrust coefficient $M_{\infty}=0.9, C_{t}$ and Mach number for a typical turbo-fan engine with a bypass ratio of about 4.5 - derived from data presented in figure 8.2 of Cumpsty and Heyes ${ }^{(19)}$.

\subsection{THE VARIATION OF ENGINE OVERALL PROPULSION EFFICIENCY}

As discussed in detail by Poll ${ }^{(1)}$ and also by Cumpsty and Heyes ${ }^{(19)}$, dimensional analysis can be used to show that $\eta_{o}$ depends only upon the ratio of the total temperature at the turbine entry, $\left(T_{o}\right)_{T E}$, to the atmospheric freestream total temperature, $\left(T_{o}\right)_{\infty}$ and the Mach number, whilst the precise form of the function depends upon the engine type, i.e.

$$
\eta_{o}=f_{5}\left(\frac{\left(T_{o}\right)_{T E}}{\left(T_{o}\right)_{\infty}}, M_{\infty}\right)
$$

The thrust coefficient, $C_{t}$, also depends upon the same parameters, i.e.

$$
C_{t}=\frac{F_{n}}{(\gamma / 2) p_{\infty} M_{\infty}^{2} A_{e}}=f_{6}\left(\frac{\left(T_{o}\right)_{T E}}{\left(T_{o}\right)_{\infty}}, M_{\infty}\right),
$$

where $F_{n}$ is the total net thrust generated and $A_{e}$ is the sum of the engine core and bypass jet exit cross-sectional areas of all the engines. Hence,

$$
\eta_{o}=f_{7}\left(C_{t}, M_{\infty}\right)
$$

The typical form of the function $f_{7}$ is given in Fig. 4. These are for a turbofan engine with a bypass ratio of about 4.5 and are taken from Appendix B of Poll ${ }^{(1)}$.

At fixed Mach number, $\eta_{o}$ exhibits a maximum at a particular value of the thrust coefficient. Consequently, in the vicinity of the maximum, $\eta_{o}$ is only weakly dependent upon $C_{t}$. The loci 
of these maximum values follow the dashed line and the variation of the maximum $\eta_{o}$ with Mach number may be represented, to a good approximation, by the relation

$$
\left(\eta_{o}\right)_{B}=\eta_{1}\left(M_{\infty}\right)^{\eta_{2}}
$$

The coefficients $\eta_{1}$ and $\eta_{2}$ depend only upon the engine type and for the engine given in Fig. 4, the values are 0.36 and 0.65 , respectively. This expression can be extended to a more general form by noting that $\eta_{2}$ depends primarily on the engine's nominal ${ }^{6}$ bypass ratio, BPR. This is demonstrated in $\operatorname{ESDU}^{(20)}$, section B3.2, figure B1, where real, though anonymised, engine data are used to show the approximate form of the relationship. Based upon these data, the expression

$$
\eta_{2}=\frac{M_{\infty}}{\left(\eta_{o}\right)_{B}} \frac{d\left(\eta_{o}\right)_{B}}{d M_{\infty}} \approx 0.70(1-0.045(B P R))
$$

is believed to be accurate to $\pm 15 \%$. Values for the nominal bypass ratio for the engines considered in this study are obtained from the $I C A O$ engine emissions data bank ${ }^{(9)}$ and are listed in Table 1.

For the engine shown in Fig. 4, the variation of overall efficiency with thrust coefficient is approximately

$$
\frac{\eta_{o}}{\left(\eta_{o}\right)_{B}} \approx 1-0.53\left(1-0.84 M_{\infty}^{2}\right)\left(\frac{C_{t}}{\left(C_{t}\right)_{B}}-1\right)^{2}+0.25\left(\frac{C_{t}}{\left(C_{t}\right)_{B}}-1\right)^{3},
$$

where the $\left(\eta_{o}\right)_{B}$ and $\left(C_{t}\right)_{\mathrm{B}}$ pairs lie on the dashed line (maximum efficiency line) given in Fig. 4. Once again, in general, the coefficients in this relation will be engine type specific. However, deviations from the values given by this expression are likely to be small.

\subsection{THE EFFECT OF MACH NUMBER ON THE DRAG POLAR WHEN THE REYNOLDS NUMBER IS CONSTANT}

Equations (4), (24)-(28) and (35)-(37) can be combined to give an approximate, but physically complete, model of the variation of $\left(\eta_{o} L / D\right)$ with the overall lift coefficient and the Mach number when the Reynolds number is constant. An example calculation, based upon a typical aircraft configuration, is presented in Fig. 5. As already noted, for any given aircraft, there is a Mach number and lift coefficient pair at which $\left(\eta_{o} . L / D\right)^{R}$ has its optimum value. In the example provided, this value is 5.86 and it occurs when $M_{o}^{R}$ is 0.835 and $\left(C_{L}\right)_{o}^{R}$ is 0.578 .

At the optimum condition,

$$
d\left(\eta_{o} L / D\right)^{R}=\frac{\partial\left(\eta_{o} L / D\right)^{R}}{\partial\left(C_{L}\right)^{R}} d\left(C_{L}\right)^{R}+\frac{\partial\left(\eta_{o} L / D\right)^{R}}{\partial M^{R}} d M^{R}=0
$$

${ }^{6}$ The term 'nominal' is necessary since the bypass ratio depends upon the engine operating condition. 


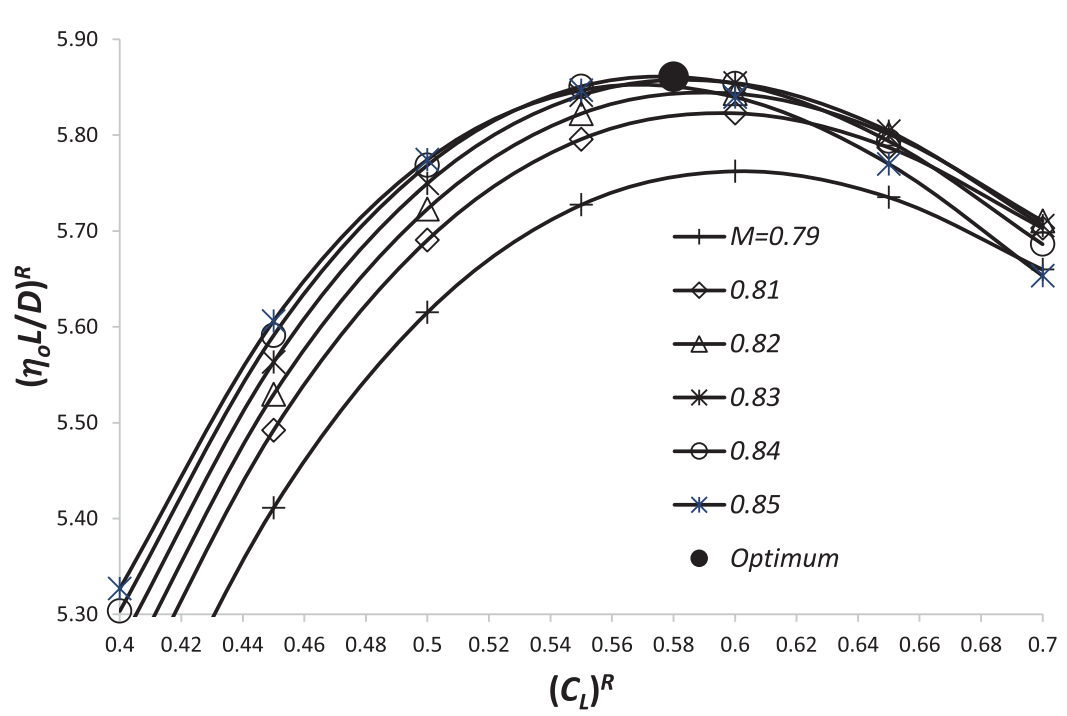

Figure 5. A typical variation of $\left(\eta_{0} L / D\right)$ with lift coefficient and Mach number at constant Reynolds number, for an aircraft with aspect ratio of 9 , sweep of $32^{\circ}, e_{L S}$ of 0.8 and $C d_{O}$ of 0.016 . The engine characteristics are taken from Fig. 4.

This equation is satisfied when the partial derivatives with respect to lift coefficient and Mach number are both zero.

When both the Reynolds number and the Mach number are constant, the engine efficiency is approximately constant and there is a value of lift coefficient at which the lift-to-drag ratio has a local maximum (or best) value and both quantities depend upon the Mach number. The condition is given by

$$
\frac{\partial\left(\eta_{o} L / D\right)^{R}}{\partial\left(C_{L}\right)^{R}} \approx \frac{\left(\eta_{o} L / D\right)^{R}}{\left(C_{L}\right)^{R}}\left(1-\frac{\left(C_{L}\right)^{R}}{C d} \frac{\partial C d}{\partial\left(C_{L}\right)^{R}}\right)=0
$$

Hence, from Equation (4), the best value of lift-to-drag ratio is

$$
\begin{aligned}
\left(\frac{L}{D}\right)_{B}^{R} \approx & \frac{1}{2}\left(1-\frac{C d_{w}}{C d_{0}}\left(1-\frac{1}{2}\left(\frac{C_{L}}{C d_{w}} \frac{\partial C d_{w}}{\partial C_{L}}\right)\right)\right) \\
& \times\left(1+\frac{C d_{w}}{C d_{0}}\left(1-\frac{C_{L}}{C d_{w}} \frac{\partial C d_{w}}{\partial C_{L}}\right)\right)^{1 / 2}\left(\frac{\pi \cdot A R \cdot e_{L S}}{C d_{o}}\right)^{1 / 2}
\end{aligned}
$$

and it occurs when

$$
\left(C_{L}\right)_{B}^{R} \approx\left(1+\frac{C d_{w}}{C d_{0}}\left(1-\frac{C_{L}}{C d_{w}} \frac{\partial C d_{w}}{\partial C_{L}}\right)\right)^{1 / 2}\left(\pi \cdot A R \cdot e_{L S} C d_{0}\right)^{1 / 2}
$$

Therefore, the best $\left(\eta_{o} . L / D\right)^{R}$ at a given Mach number is approximately equal to the product of $\eta_{o}$, from Equation (35) and the corresponding best $(L / D)^{R}$ from Equation (40). 


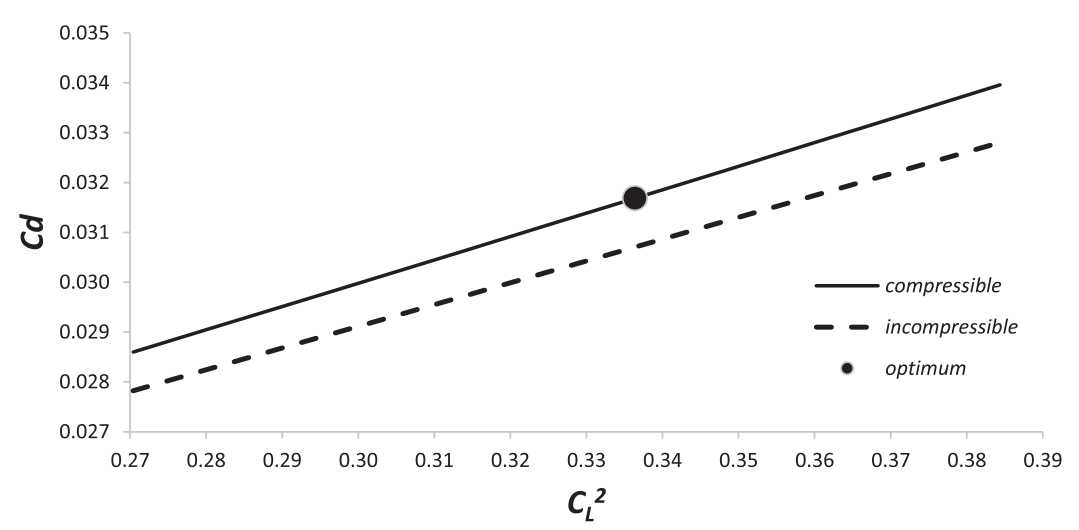

Figure 6. A comparison between the high-speed drag polar at the Mach number for optimum $\left(\eta_{o} L / D\right)^{R}$ and low-speed drag polar for the aircraft used in Fig. 5.

The value of the Mach number at which the optimum condition occurs comes from a solution of

$$
\frac{\partial\left(\eta_{o} L / D\right)^{R}}{\partial M^{R}}=\frac{\left(\eta_{o} L / D\right)^{R}}{M^{R}}\left(\frac{M^{R}}{\eta_{o}} \frac{d \eta_{o}}{d M^{R}}-\frac{M^{R}}{C d} \frac{\partial C d}{\partial M^{R}}\right)=0,
$$

i.e. when there is a balance between the rate of wave drag rise with increasing Mach number, driving $(L / D)$ down, and the rate of engine overall efficiency increase with increasing Mach number, driving $\eta_{o}$ up. This is achieved when

$$
\frac{M^{R}}{C d} \frac{\partial C d_{w}}{\partial M^{R}}=\eta_{2}
$$

Therefore, from Equation (36), in addition to the aspect ratio, sweep angle, $e_{L S}$ and $C d_{o}$, the engine bypass ratio, $B P R$, is also a factor determining the optimum condition.

Continuing with the example, the variation of $C d$ with $C_{L}{ }^{2}$ when the Mach number has the optimum value is shown in Fig. 6 . Despite the complexity of the calculation, $C d$ still exhibits an almost linear variation with $C_{L}{ }^{2}$ and a 'least-squares' fit gives

$$
C d \approx 0.0159+0.0470 C_{L}^{2}
$$

However, in the absence of wave drag, the polar Equation (4) is

$$
C d=\left(C d_{0}\right)_{M=0.5}+\left(\frac{1}{\pi \cdot A R \cdot e_{L S}}\right) C_{L}^{2}=0.0160+0.0437 C_{L}^{2}
$$

Therefore, when the lift coefficient is also at its optimum value $\left(C_{L}=0.578\right)$, relative to Equation (45), $C d$ has risen by 10 drag counts $(\approx 3.3 \%$ increase) and the gradient of the polar has grown by about $0.0033(\approx 7.5 \%$ increase $)$. However, the values of $C d_{0}$ are almost the same. This suggests that the zero-lift, profile drag coefficient is essentially unchanged by the development of wave drag $(<1 \%)$. This insensitivity, whilst perhaps surprising, is consistent with the assumption made in Equation (4) and with the Obert data given in Fig. 1. 
Therefore, according to the Shevell model, the principal effect of wave drag at the conditions for optimum $\left(\eta_{o} L / D\right)^{R}$ is to decrease the value of the Oswald factor relative to its low-speed value.

When the Mach number is equal to $M_{o}^{R}$, Equation (4) becomes

$$
C d \approx C d_{0}+\left(\frac{e_{L S}}{e_{o}}\right)\left(\frac{1}{\pi \cdot A R \cdot e_{L S}}\right) C_{L}^{2}
$$

Using this relation in Equation (39) to obtain $(L / D)_{\mathrm{o}}$ and also using Equation (35) gives

$$
\begin{gathered}
\left(\eta_{o} L / D\right)_{o}^{R} \approx \frac{\left(\eta_{o}\right)_{o}^{R}}{2}\left(\pi \cdot A R \cdot e_{L S}\left(\frac{e_{o}}{e_{L S}}\right)\right)^{1 / 2}\left(\frac{1}{C d_{0}}\right)^{1 / 2}, \\
\left(\frac{L}{D}\right)_{o}^{R} \approx \frac{1}{2}\left(\pi \cdot A R \cdot e_{L S}\left(\frac{e_{o}}{e_{L S}}\right)\right)^{1 / 2}\left(\frac{1}{C d_{0}}\right)^{1 / 2}
\end{gathered}
$$

and

$$
\left(C_{L}\right)_{o}^{R} \approx\left(\pi \cdot A R \cdot e_{L S} \cdot\left(\frac{e_{o}}{e_{L S}}\right)\right)^{1 / 2} C d_{0}{ }^{1 / 2}
$$

For consistency between Equations (40) and (41) and (48) and (49), at the optimum condition,

$$
\left(\frac{C_{L}}{C d_{w}} \frac{d C d_{w}}{d C_{L}}\right)_{o}^{R}=2
$$

and, hence, from Equations (41) and (49)

$$
\frac{e_{o}}{e_{L S}} \approx 1-\left(\frac{C d_{w}}{C d_{0}}\right)_{o}^{R}
$$

Whilst this is a very simple result, it is a consequence of the definition of 'wave drag' as used in the Shevell model, i.e. according to Equations (4), and Equation (51) may not hold if the wave drag is defined in a different way.

Calculations have been carried out for the two loading distributions shown in Fig. 3 for aircraft with aspect ratios in the range of 7.5-10 and $C d_{o}$ lying between 0.014 and 0.02 . The results show that, when the Mach number is at $M_{o}^{R}, C d_{0}$ varies by a maximum of $2 \%$. In addition, the increase in the lift-dependent drag coefficient due to wave drag is given, to an accuracy of $\pm 10 \%$, by the expression

$$
\left(\frac{d C d_{w}}{d C_{L}^{2}}\right)_{o}^{R}=\frac{\delta_{3}}{\pi \cdot A R} \approx 0.167\left(\eta_{2} e_{L S}\right)
$$

Hence, the total lift-dependent drag coefficient may be written as

$$
\left(\frac{1}{\pi \cdot A R \cdot e_{o}}\right) \approx\left(\left(\frac{1+\delta_{1}+\delta_{2}+\delta_{3}}{\pi \cdot A R}\right)+k_{1}\right)
$$




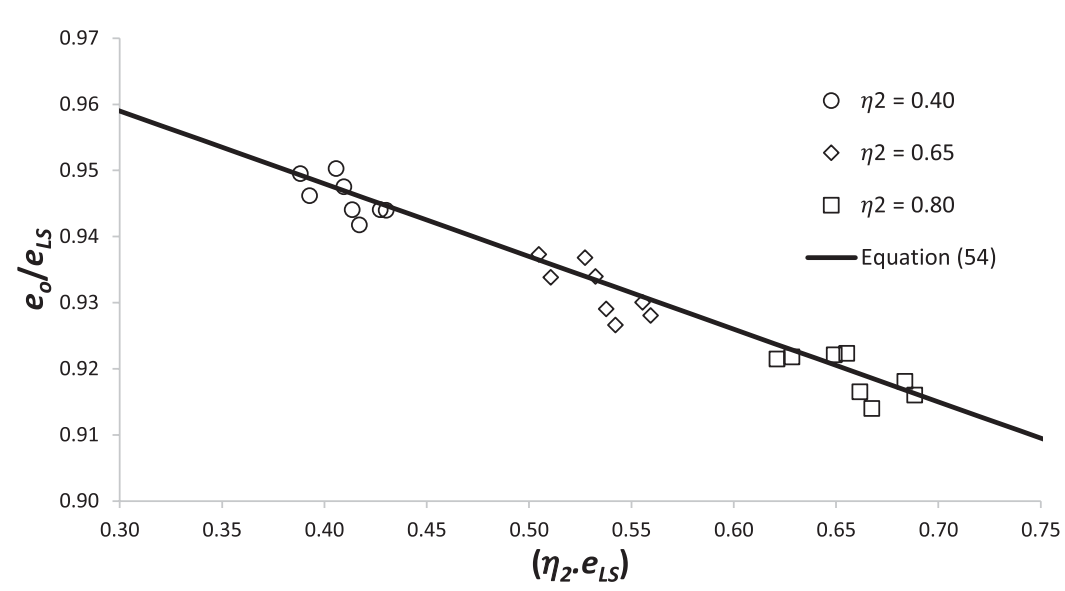

Figure 7. The variation of the ratio of the high-speed and low-speed Oswald factors, $e_{0} / e_{L S}$, with the product of $\eta_{2}$ and the low-speed Oswald factor, $e_{L S}$.

Taking typical values, the contributions of the various elements to the total lift-dependent drag coefficient are approximately $80 \%$ from the wing vortex system, $15 \%$ from the profile and trim drags and $5 \%$ from the wave drag. Since the contribution from wave drag is so small, a relatively crude estimate does not introduce any significant inaccuracy into the overall estimation process. Therefore, using more sophisticated methods to determine wave drag, e.g. CFD, would greatly increase the complexity of the calculation, but would have little impact upon the result. The largest uncertainty in the estimate is still that associated with the value of $k_{1}$.

Furthermore, curve fitting the data suggests that the relation

$$
\frac{e_{o}}{e_{L S}} \approx 0.992-0.11\left(\eta_{2} e_{L S}\right)
$$

is accurate to $\pm 0.5 \%$. This relation is shown in Fig. 7 .

Finally, by combining Equations (51) and (54), the wave drag coefficient at the optimum condition is

$$
\left(C d_{w}\right)_{o}^{R} \approx 0.11\left(\eta_{2} e_{L S}\right) C d_{0},
$$

whilst, to within $\pm 2 \%$, the total drag coefficient is given by

$$
(C d)_{o}^{R} \approx 1.99 C d_{0}
$$

The calculations also show that the Mach number at which the optimum occurs, $M_{o}^{R}$, exhibits a very weak dependence upon $C d_{0}$. This is consistent with the assumption made in Ref. 2 that $M_{o}^{R}$ is independent of $C d_{0}$ and, hence, Reynolds number. However, $M_{o}^{R}$ does have some dependence on $\eta_{2}$, i.e.

$$
\frac{\partial M_{o}^{R}}{\partial \eta_{2}} \approx 0.06
$$


Therefore, if an aircraft's engines are replaced with ones having a different bypass ratio, the optimum Mach number for the new combination will be slightly different.

\subsection{THE CHARACTERISTIC PARAMETERS}

It is now possible to write down expressions for all the $\psi$ parameters. As already shown, $\psi_{0}$ comes from Equation (23) and depends only upon aircraft geometry. In principle, $\psi_{1}$ can be obtained from Equation (47). However, since there is no simple, accurate method available for the estimation of $\eta_{o}$, at this stage in the development of the method $\Psi_{1}$ is best obtained from empirical data. This process is described in detail in the next section.

The value of $\tau$ comes from Equations (8), (23)-(26) and (54), i.e.

$$
\tau=-\left(\frac{C_{F}^{a c}}{e_{o}}\right)\left(\frac{d e_{o}}{d C_{F}^{a c}}\right) \approx \frac{\left(0.992-0.22\left(\eta_{2}\left(e_{L S}\right)_{o}\right)\right)}{\left(0.992-0.11\left(\eta_{2}\left(e_{L S}\right)_{o}\right)\right)}\left(\pi \cdot A R \cdot k_{1} \cdot\left(e_{L S}\right)_{o}\right)
$$

In this analysis, $\tau$ is a constant. Therefore, in Equation (58), $e_{L S}$ should be evaluated at the optimum Reynolds number for a value of the aircraft mass that is near the middle of the operating range. Sample estimates for the aircraft listed in Table 1 show that this requirement is satisfied if $m / M T O M$ is taken to be 0.8 , see Equation (16). The corresponding value of $E_{o}$ is given by

$$
E_{o}=\left(0.992-0.11 \eta_{2}\left(e_{L S}\right)_{o}\right)\left(e_{L S}\right)_{o}\left(\left(C_{F}^{a c}\right)_{o}\right)^{\tau}
$$

The parameter $\psi_{2}$ can now be determined by combining Equations (8), (10), (23) and (49), i.e.

$$
\psi_{2} \approx\left(\pi \cdot A R \cdot \psi_{0} \cdot E_{o}\right)^{1 / 2}
$$

Similarly, $\psi_{3}$ comes from Equations (8), (11), (23) and (48), i.e.

$$
\psi_{3} \approx \frac{1}{2}\left(\frac{\pi \cdot A R \cdot E_{o}}{\psi_{0}}\right)^{1 / 2}
$$

As shown in Poll ${ }^{(1)}$, estimates of $\left(\eta_{o} L / D\right)$ are sensitive to $\psi_{4}$ and therefore this quantity needs to be determined as accurately as possible. As indicated above, in principle, $\psi_{4}$, which is equal to $M_{o}^{R}$ - see Equation (12), can be calculated directly from the wave drag model. However, since the Shevell method is highly simplified, the absolute accuracy of such an estimate is unknown. Therefore, in the first instance, an alternative approach is required.

The most accurately known speed is the maximum operational Mach number, $M_{M O}$, since this is specified in the TCDS. In addition, the long-range cruise Mach number, $M_{L R C}$, is often to be found in either the aircraft $A P R$, or other standard reference sources, e.g. Jane's ${ }^{(7)}$. Figure 8 shows the variation of $M_{L R C}$ with $M_{M O}$ for those aircraft in Table 1 for which $M_{L R C}$ is known. There is a strong correlation between the two quantities and taking $M_{L R C}$ to be $0.935\left(M_{M O}\right)$ gives a maximum error of about $4 \%$. This relation can then be extended to provide an estimate of the maximum range cruise Mach number, $M_{M R C},\left(=M_{o}\right)$ by noting that, from equation (24) of Poll ${ }^{(1)}, M_{L R C}$ is approximately 1.035 times $M_{M R C}$. Hence, 


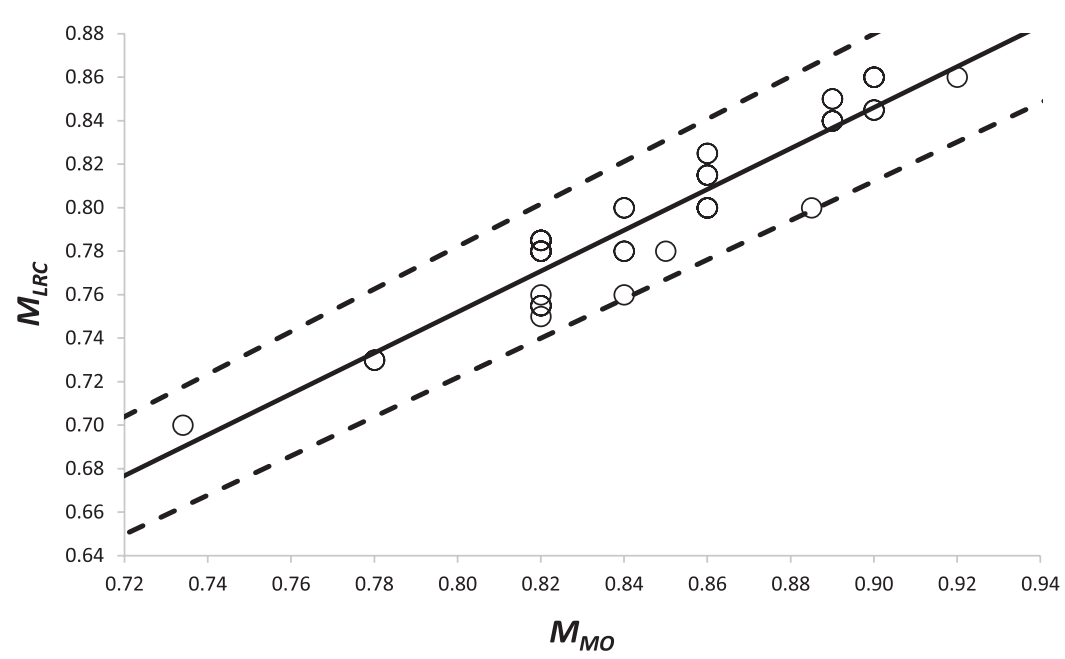

Figure 8. The relationship between the long-range cruise Mach number, $M_{L R C}$, and the maximum operational Mach number, $M_{M O}$. The solid line is given by $M_{L R C}$ equal to $0.940 M_{M O}$, and the dashed lines represent $\pm 4 \%$ variation.

$$
\psi_{4}=M_{o}=M_{M R C} \approx \frac{M_{L R C}}{1.035} \approx 0.910 M_{M O}
$$

To minimise the error, if the long range cruise Mach number is accurately known, $\psi_{4}$ is taken to be $M_{L R C}$ divided by 1.035 . However, If $M_{L R C}$ is not available, then Equation (62) should be used. Estimated values of $\psi_{4}$ are given in Table 2.

The parameter $\psi_{5}$ in Equation (14) is the non-dimensional, wing reference area. Unlike the plan area of the exposed, or 'wetted', part of the wing, which is easily and unambiguously obtained from a general arrangement drawing, the aerodynamic reference area, $S_{r e f}$, must be given a clear definition. This is because the 'reference' area includes part of the wing that is covered by the fuselage. In this analysis, $S_{\text {ref }}$ is defined as the plan area of the exposed wing plus the area of the rectangle inside the fuselage whose vertices are the points where the leading and trailing edges meet the fuselage $\operatorname{side}^{7}$. The values of $S_{\text {ref }}$ are listed in Table 1, and the corresponding values of $\psi_{5}$ are given in Table 2.

Finally, the parameter $\psi_{6}$ in Equation (15) is the non-dimensional, maximum take-off mass. However, whilst a given aircraft type will have a fixed external geometry and specified engines, its MTOM is not necessarily fixed. This is because the manufacturer usually offers a number of 'weight variants' to satisfy different airline requirements ${ }^{8}$. Therefore, the MTOM may have a range of values, with the largest being as much as $25 \%$ greater than the smallest. For a particular aircraft, the weight variant can usually be found from the tail number. Table 1 contains a reference $M T O M$ and the corresponding weight variant number for each

${ }^{7}$ This is the Airbus definition. However, there are a number of other definitions in use.

${ }^{8}$ This is because several of the operational costs levied upon the airlines are determined by the aircraft maximum take-off weight. 
aircraft, and the corresponding values of $\left(\psi_{6}\right)_{\text {ref }}$ are given in Table 2 . The reference value can be converted to cover other aircraft by introducing a weight variant factor, $F_{w v}$, i.e.

$$
\psi_{6}=F_{w v}\left(\psi_{6}\right)_{r e f},
$$

where $F_{w v}$ is the ratio of the actual $M T O M$, as given either in the type certificate, or the relevant $A P R$, to the $M T O M$ of the reference aircraft.

\subsection{THE ESTIMATION OF $\psi_{1}$ FROM THE PAYLOAD-RANGE DIAGRAM}

$A P R s$ usually contain a number of payload-range diagrams whose construction requires a knowledge of $\left(\eta_{o} L / D\right)$. Consequently, with suitable interpretation, these diagrams can be used to provide estimates of $\left(\eta_{o} L / D\right)_{o}$ under specified conditions and, hence, values of $\Psi_{l}$ can be established.

At the beginning of the take-off run (brakes-off point), the take-off mass, TOM, can be broken down into a number of components, i.e.

$$
T O M=O E M+P M+F M_{n c}+T F M=Z F M+F M_{n c}+T F M
$$

Here $P M$ is the payload mass, TFM is the mass of the fuel that will be used during the trip, $F M_{n c}$ is the mass of the fuel that is not consumed during the flight and $O E M$ is the operational empty mass. The $O E M$ is the mass of the aircraft before any payload and fuel are loaded and is given by

$$
O E M=M Z F M-M P M
$$

where $M Z F M$ is the maximum permitted zero-fuel mass (given in the aircraft type certificate) and $M P M$ is the maximum payload. The $M Z F M$ and typical values of the $M P M$ are usually given in the aircraft's $A P R$.

As described by Poll ${ }^{(1)}$, the trip fuel mass is related to the take-off mass by

$$
\frac{T F M}{T O M}=\alpha_{t} \approx 1-E X P\left(-\left(X_{t}+\varepsilon_{t}\right)\right)
$$

where $X_{t}$ is the non-dimensional trip distance, given by

$$
X_{t}=\frac{g R_{t}}{\left(\eta_{o} L / D\right)_{o} L C V},
$$

with $R_{t}$ being the great circle distance between the departure and destination points, $\varepsilon_{t}$ the total 'lost fuel' index and $L C V$ the lower calorific value of the fuel. The total lost fuel index captures the additional fuel used in the climb and descent phases, any fuel wasted by not cruising at the optimum speed and height, any fuel used to fly extra distance due to route deviations and fuel lost, or saved, because of the wind. However, for a maximum range cruise in still air

$$
\varepsilon_{t}=\left(\varepsilon_{c d}\right)_{\text {min }}+\left(\frac{1-n}{n}\right) X_{t},
$$


where $\varepsilon_{c d}$ is the sum of the lost fuel indices for the climb and descent phases and $n$ is given by

$$
n=\frac{\left(\eta_{o} L / D\right)_{a v g}}{\left(\eta_{o} L / D\right)_{o}}
$$

In general, the parameter $n$ depends upon the flight trajectory, i.e. the profile of Mach number versus altitude, and the aircraft's mass at the beginning of the cruise.

The non-consumed fuel, some of which may be used on subsequent flights, must be greater than, or equal to, the minimum reserve fuel, $F M_{\text {res }}$, as required by the regulatory authority, plus any extra fuel, $F M_{\text {ext }}$, specified by the operator, or the aircraft captain. However, for a maximum range flight with a given amount of trip fuel, the non-consumed fuel must also have its minimum possible value and so

$$
\left(F M_{n c}\right)_{\min }=\left(F M_{r e s}\right)_{\min }=\beta_{\min } T O M
$$

Hence, from Equations (64), (66) and (70),

$$
\alpha_{t}=1-\beta_{\min }-\frac{Z F M}{T O M}
$$

and, on the payload-range diagram, the variation of zero-fuel mass with range for a given take-off mass is given by

$$
\frac{Z F M}{T O M}=E X P\left(-\left(\left(\varepsilon_{c d}\right)_{\min }+\frac{X_{t}}{n}\right)\right)-\beta_{\text {min }}
$$

In Appendix C, it is shown that, under EU Ops rules (see Airbus ${ }^{(23)}$ ), the reserve fuel requirement can always be expressed in the form

$$
\beta_{\text {min }} \approx 0.05 \alpha_{t}+\lambda\left(1-\alpha_{t}\right)=\frac{(0.05+(\lambda-0.05)(Z F M / T O M))}{(1.05-\lambda)},
$$

where

$$
\lambda=\left(\left(\varepsilon_{c d}\right)_{\min }+\frac{\left(X_{a d}+X_{d a}+X_{f r}\right)}{n}\right)
$$

Here, $X_{a d}, X_{d a}$ and $X_{f r}$ are the non-dimensional, additional cruise distances that can be flown using the 'additional', 'diversionary airport' and 'final reserve' elements of the reserve fuel. Combining Equations (72) and (73) gives

$$
\frac{Z F M}{T O M}=\left(\frac{1.05-\lambda}{E X P\left(\varepsilon_{c d}\right)_{\min }}\right) E X P\left(-\frac{X_{t}}{n}\right)-0.05
$$

This equation is the tool that is used to extract data from the payload range diagram and, to check the accuracy of the results, two implementation techniques have been used. 


\subsection{Method 1}

On the payload-range diagram, each point represents a complete flight. If the aircraft is always operating at optimum $\left(\eta_{o} L / D\right)$, the appropriate cruise altitude and, hence, the Reynolds number is determined by the aircraft mass, $m$ - see Equation (20). This being the case, $\left(\eta_{o} L / D\right)_{o}$ also varies with $m$ - see Equation (21). The value of $\left(\eta_{o} L / D\right)_{o}$ at the beginning of the cruise is determined, primarily, by the take-off mass and, as the aircraft consumes fuel, both $m$ and $\left(\eta_{o} L / D\right)_{o}$ decrease. However, since the variation is usually small, to a good approximation, a complete flight may be characterised by the average value of the mass in cruise.

As noted in Ref. 1, at the start of cruise, the mass is about $97.5 \%$ of TOM and, at the end, it is roughly $101 \%$ of the landing mass, $L M^{9}$. Therefore, using Equations (66)-(68) and taking $\left(\varepsilon_{c d}\right)_{\min }$ to be $0.0067-$ see Poll $^{(1)}$,

$$
\frac{m_{\text {avg }}}{T O M}=\frac{\left(m_{i c}+m_{f c}\right)}{2(T O M)} \approx \frac{1}{2}\left(1.985-1.01 \frac{T F M}{T O M}\right) \approx 0.99\left(1.0-0.51 \frac{X_{t}}{n}\right)
$$

and, from Equations (21), (67) and (69), for flight in the International Standard Atmosphere,

$$
\frac{X_{t}}{n}=\frac{g R_{t}}{\left(n \psi_{1}\right) L C V}\left(\left(\frac{a}{\psi_{5}^{b}}\right)\left(\psi_{7}\left(\frac{M T O M}{T O M}\right)\left(\frac{T O M}{m_{\text {avg }}}\right)\right)^{b \iota_{o} \kappa_{o}}\right)^{\left(\frac{1+\tau}{2}\right)}
$$

Substituting the results from Equation (76) into Equation (77), neglecting terms that are small in comparison with unity and rearranging gives

$$
\frac{X_{t}}{n} \approx\left(\frac{\psi_{8} g R_{t}}{n \psi_{1} L C V}\right)\left(1+0.51\left(b \iota_{o} \kappa_{o}\right)\left(\frac{1+\tau}{2}\right)\left(\frac{\psi_{8} g R_{t}}{n \psi_{1} L C V}\right)\right),
$$

where

$$
\psi_{8}=\frac{1}{G_{5}}\left(\left(\frac{a}{\psi_{5}^{b}}\right)\left(\frac{\psi_{7}}{0.99}\left(\frac{\text { MTOM }}{\text { TOM }}\right)\right)^{b \iota_{o} \kappa_{0}}\right)^{\left(\frac{1+\tau}{2}\right)}
$$

Hence, by substituting the result from Equation (78) into Equation (75) and expanding the exponential function as a power series gives

$$
\begin{aligned}
\frac{Z F M}{T O M}+0.05= & \left(\frac{1.05-\lambda}{\operatorname{EXP}\left(\left(\varepsilon_{c d}\right)_{\min }\right)}\right)\left(1-\left(\frac{\psi_{8} g R_{t}}{n \psi_{1} L C V}\right)\right. \\
& \left.+\frac{1}{2}\left(1-1.01\left(b \iota_{o} \kappa_{o}\right)\left(\frac{1+\tau}{2}\right)\right)\left(\frac{\psi_{8} g R_{t}}{n \psi_{1} L C V}\right)^{2}-\ldots\right)
\end{aligned}
$$

For a given aircraft, $\left(\varepsilon_{c d}\right)_{\min }$ is fixed and, as argued in Ref. 1, its value is expected to be approximately $0.0067 \pm 0.0026$. Therefore, for any particular value of TOM, if the reserve

${ }^{9}$ Since the trip fuel includes the fuel used in climb and descent, the smallest value that TFM/TOM can have is 0.0348 . In this case, since there is no cruise phase, no fuel is consumed in the cruise. 


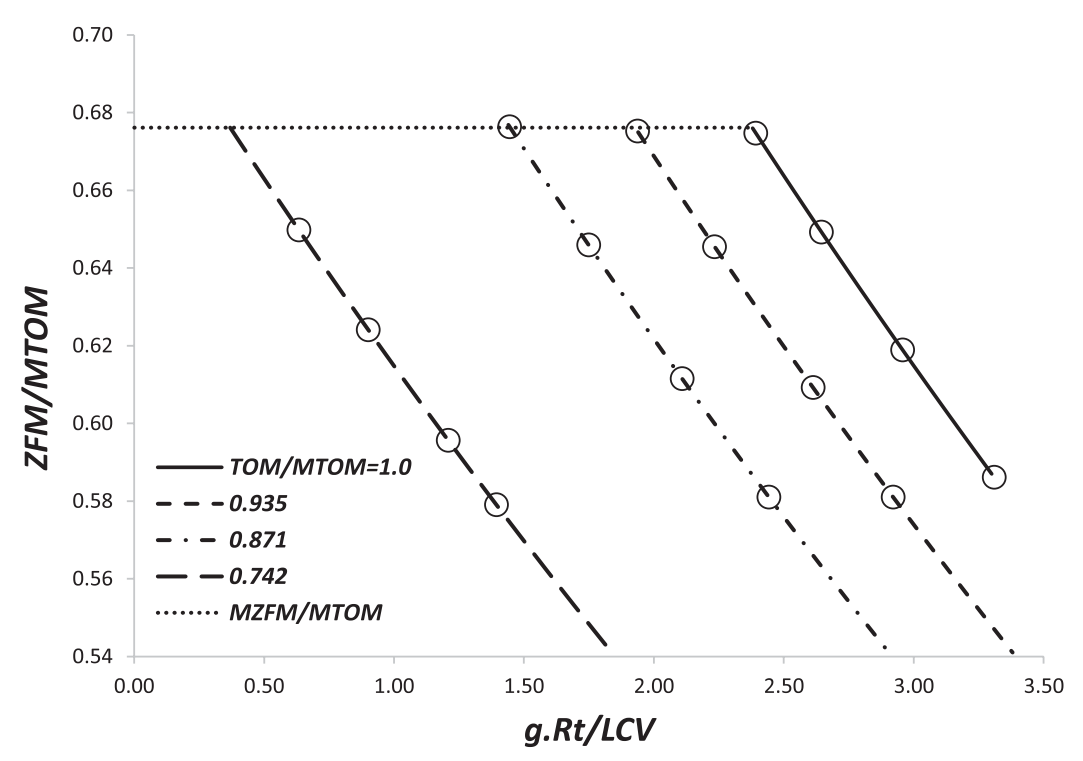

Figure 9. The payload-range diagram for the Boeing 777-300ER. Lines of constant take-off mass are constructed using the estimates, based upon method 1, for $\left(n \Psi_{1}\right)$ and $\lambda$ and the circles are the digitised values taken from the manufacture's payload-range diagram. The values of $\left(n \Psi_{1}\right)$ and are $0.320 \pm 0.001$ and $0.030 \pm 0.003$ respectively.

fuel coefficient, $\lambda$, the distance flown, $R_{t}, \psi_{8}$ and the zero-fuel mass, $Z F M$, are known, the value of $\left(n \Psi_{1}\right)$ can be deduced. However, whilst $R_{t}$ and $Z F M$ may be read directly from the payload-range diagram, $\lambda$ is usually not known 'a priori'. Consequently, a calculation scheme is required to determine the combined values of $\left(n \Psi_{1}\right)$ and $\tau$ that best describe the given variation of ZFM with $R_{t}$ at fixed TOM.

To test the robustness of the results, two independent numerical schemes have been used. Method 1 involved the inversion of Equation (80) and, with $\lambda$ fixed, using known values of $Z F M$ and $R_{t}$ taken along a line of constant TOM to determine $\left(n \Psi_{l}\right)$ directly. The value of $\lambda$ was then varied until the 'least squares' linear fit of $\left(n \Psi_{l}\right)$ against $R_{t}$ had a zero slope, i.e. both $\left(n \Psi_{l}\right)$ and $\lambda$ had single values for the whole dataset. An example showing the success of the fitting process is given in Fig. 9.

It was found that each payload-range diagram only yielded a single value of $\left(n \Psi_{1}\right)$ and the variations in the values of both $\left(n \Psi_{1}\right)$ and for different values of TOM/MTOM were generally small. The values of $\left(n \Psi_{1}\right)$ were not sensitive to the choice of $\left(\varepsilon_{c d}\right)_{\min }$. However, when the range parameter was small, $\left(n \Psi_{l}\right)$ was found to be very sensitive to errors in ZFM/TOM. These observations are all consistent with the behaviour implicit in Equation (80). The accuracy demonstrated in Fig. 9 was not achieved in all cases. However, the larger discrepancies were found to be due, primarily, to inaccuracies and inconsistencies in the payload-range diagrams given in some $A P R s$.

\subsection{Method 2}

For method 2, it was assumed that the Reynolds number effects were small and, using the same values of $Z F M$ versus $R t$ at fixed TOM, Equation (75) was solved numerically to determine pairs of values of $\left(\eta_{o} L / D\right)_{a v g}$ and $\lambda$ for which the data fit had the minimum least square root of the sum of deviations (Appendix D). The values of $\left(\eta_{o} L / D\right)_{\text {avg }}$ were then converted to $\left(n \Psi_{l}\right)$ 


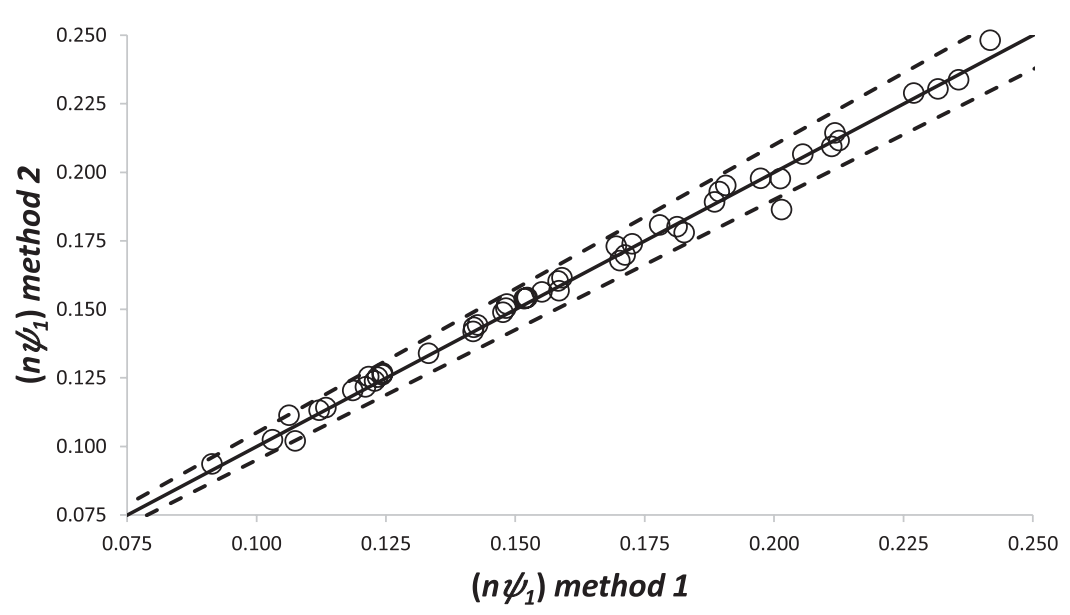

Figure 10. A comparison between the values of $\left(n \Psi_{1}\right)$ as determined by analysis methods 1 and 2 . The solid line has unit slope and the dashed lines indicate $a \pm 5 \%$ variation.

by using Equations (21) and (67), with the mid-range value being taken for $R_{t}$. The results from the two methods for all the aircraft considered in this study are compared in Fig. 10. The values obtained from the two methods were found to have a maximum difference of $5 \%$ and a mean difference of $1.5 \%$. The 'best estimate' for $\left(n \Psi_{l}\right)$ was taken to be the average of the two values.

The $\left(\eta_{o} L / D\right)_{a v g}$ values obtained by method 2 can also be compared directly with those given in other references, e.g. Martinez-Val et al. ${ }^{(21)}$ and Randle et al. ${ }^{(22)}$. In both of those studies, the authors sought to estimate $\left(\eta_{o} L / D\right)_{\text {avg }}$ either from payload-range diagrams or from flight data recorder information, and results are provided for 20 of the aircraft listed in Table 1. These are compared with the method 2 values in Fig. 11. The Martinez-Val et al. results exhibit greater scatter than those of Randle et al. and, whilst some of this is attributable to reading errors due to the small size of the figures in both references, the general level of agreement is reasonable and reflects the sensitivity of the estimates to small errors in the input data.

Finally, to obtain $\Psi_{1}$, it is necessary to estimate the value of $n$; see Equation (69). This quantity may be expressed as

$$
n=\left(\frac{\left(\eta_{o} L / D\right)_{I C}}{\left(\eta_{o} L / D\right)_{o}}\right)\left(\frac{\left(\eta_{o} L / D\right)_{a v g}}{\left(\eta_{o} L / D\right)_{I C}}\right)
$$

where all the terms are evaluated at the same Reynolds number. The first bracketed term is given by Equation (22) and depends upon $M_{\infty} / M_{\mathrm{o}}$ and the ratio of the lift coefficient at the beginning of cruise, $\left(C_{L}\right)_{I C}$, to the optimum value, $\left(C_{L}\right)_{o}$. Payload-range diagrams are usually based on a fixed take-off mass, a fixed Mach number and either a fixed altitude or a step-climb profile. In all the cases considered in this analysis, the diagram was based upon the long-range cruise Mach number. Therefore, by definition, at the start of the cruise

$$
\frac{\left(\eta_{o} L / D\right)_{I C}}{\left(\eta_{o} L / D\right)_{o}} \approx 0.99
$$




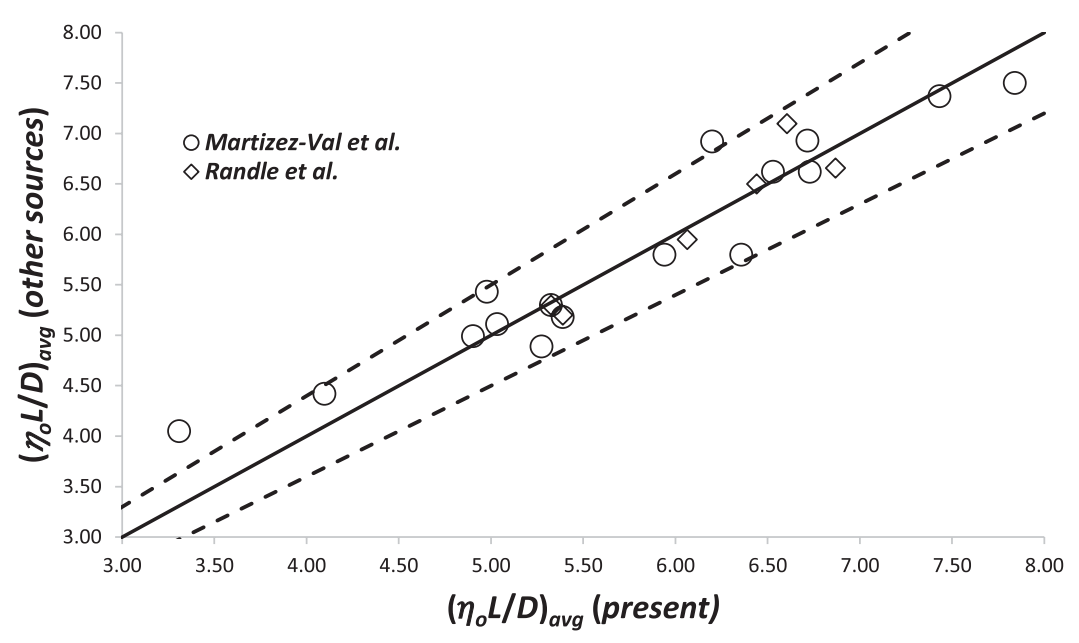

Figure 11. A comparison between the estimated values of $\left(\eta_{o} L / D\right)_{\text {avg }}$ and the previously published results of Martinez-Val et al. ${ }^{(21)}$ and Randle et al. ${ }^{(22)}$. The solid line has unit slope, and the dashed lines indicate $a \pm 10 \%$ variation.

If the aircraft were then to follow a cruise-climb trajectory, the ratio of $\left(\eta_{o} L / D\right)$ to its optimum value would stay at this value. However, at present, to guarantee safe separation, aircraft are not allowed to cruise climb, although, if circumstances permit, an occasional step change to a higher cruise altitude may be possible. Therefore, the second bracketed term provides a further correction to allow for the additional fuel required when following constant-altitude or step-climb trajectories. As shown in Ref. 1, if the flight trajectory is known, this term can be evaluated exactly. However, examination of the complete solution suggests that, in current operating conditions, this term is likely to have a value in the region of 0.985 . Hence,

$$
n \approx 0.975
$$

With the value of $n$ determined, $\Psi_{l}$ can be estimated and the values are listed in Table 2.

\subsection{THE ESTIMATION OF $\eta_{o}$, (L/D) AND TOTAL THRUST}

From Equations (12) and (35),

$$
\frac{\left(\eta_{o}\right)_{B}^{R}}{\left(\eta_{o}\right)_{o}^{R}}=\left(\frac{M_{\infty}}{\psi_{4}}\right)^{\eta_{2}}
$$

Since $\eta_{o}$ depends only upon Mach number, this result can be combined with the function $f_{1}$ from Equations (A-14), or (A-15) to give

$$
\frac{(L / D)_{B}^{R}}{(L / D)_{o}^{R}}=\frac{\left(\eta_{o} L / D\right)_{B}^{R}}{\left(\eta_{o} L / D\right)_{o}^{R}}\left(\frac{\left(\eta_{o}\right)_{o}^{R}}{\left(\eta_{o}\right)_{B}^{R}}\right)=f_{1}\left(\frac{\psi_{4}}{M_{\infty}}\right)^{\eta_{2}}
$$


Hence, at the optimum condition, using Equations (11), (17) and (19) and ignoring products of small quantities,

$$
(L / D)_{o} \approx G_{7} \psi_{3}\left(\left(\frac{\psi_{5}^{b}}{a}\right)\left(\frac{1}{\psi_{7}}\left(\frac{m}{M T O M}\right)\right)^{b \iota_{o} \kappa_{o}}\right)^{\left(\frac{1+\tau}{2}\right)}
$$

where

$$
G_{7}=\left(f_{1}\right)_{o}\left(\frac{1}{1+\varepsilon}\right)^{\eta_{2}}\left(\frac{1}{G_{3}}\right)^{\left(\frac{1+\tau}{2}\right)}=\left(\frac{1}{1+\varepsilon}\right)^{\eta_{2}} G_{5}
$$

It follows immediately from Equations (21) and (86) that

$$
\left(\eta_{o}\right)_{o}=\frac{\left(\eta_{o} L / D\right)_{o}}{(L / D)_{o}}=\left(\frac{\psi_{1}}{\psi_{3}}\right)(1+\varepsilon)^{\eta_{2}}=\left(\frac{\psi_{1}}{\psi_{3}}\right)\left(\frac{M_{o}}{\psi_{4}}\right)^{\eta_{2}}
$$

Hence, from Equation (35), the engine constant $\eta_{1}$ is given by

$$
\eta_{1}=\left(\frac{\psi_{1}}{\psi_{3}}\right)\left(\frac{1}{\psi_{4}}\right)^{\eta_{2}}
$$

The values of $\eta_{1}$ are included in Table 2 .

As shown in Poll ${ }^{(1)}$, provided the engines are sized such that $L / D$ and $\eta_{o}$ both reach maximum values at the same conditions in straight and level flight, then

$$
\frac{C_{t}}{\left(C_{t}\right)_{B}} \approx \frac{C_{L}}{\left(C_{L}\right)_{B}}
$$

and, assuming that Equation (37) is approximately the same for all engine types,

$$
\frac{\eta_{o}}{\left(\eta_{o}\right)_{B}} \approx 1-0.53\left(1-0.84 M_{\infty}^{2}\right)\left(\frac{C_{L}}{\left(C_{L}\right)_{B}}-1\right)^{2}+0.25\left(\frac{C_{L}}{\left(C_{L}\right)_{B}}-1\right)^{3}
$$

In addition, Poll and Schumann ${ }^{(2)}$ have shown that, when the mass is fixed,

$$
\frac{C_{L}}{\left(C_{L}\right)_{B}} \approx\left(\frac{C_{L}}{\left(C_{L}\right)_{o}}\right) \frac{\left(C_{L}\right)_{o}}{\left(C_{L}\right)_{B}}=f_{4}\left(\frac{C_{L}}{\left(C_{L}\right)_{o}}\right)^{v},
$$

where $f_{4}$ and $v$ are given in Appendix A. Therefore, the overall engine efficiency for any combination of Mach number and lift coefficient within the range of validity of the basic relations set out in Appendix A, is given by

$$
\begin{aligned}
\frac{\eta_{o}}{\left(\eta_{o}\right)_{o}} \approx & \left(\frac{M_{\infty}}{M_{o}}\right)^{\eta_{2}}\left(1-0.53\left(1-0.84 M_{\infty}^{2}\right)\left(\left(f_{4}\left(\frac{C_{L}}{\left(C_{L}\right)_{o}}\right)^{v}\right)-1\right)^{2}\right. \\
& \left.+0.25\left(\left(f_{4}\left(\frac{C_{L}}{\left(C_{L}\right)_{o}}\right)^{v}\right)-1\right)^{3}\right) .
\end{aligned}
$$


Furthermore, the combination of Equations (22), (86) and (93) allows the estimation of $(L / D)$ for an aircraft of given mass to be determined for any combination of Mach number and lift coefficient, i.e.

$$
(L / D) \approx \frac{\left(\eta_{o} L / D\right)}{\left(\eta_{o} L / D\right)_{o}} \frac{\left(\eta_{o}\right)_{o}}{\left(\eta_{o}\right)}(L / D)_{o}
$$

In straight and level flight, the thrust is equal to the drag and the lift is equal to the aircraft weight and so the total engine thrust, $F_{n}$, is given by

$$
F_{n} \approx \frac{m g}{(L / D)}
$$

\subsection{SENSITIVITY TO UNCERTAINTY IN THE INPUT VALUES}

The overall accuracy of the method may be judged by examining the sensitivity of $\left(\eta_{o} L / D\right)$ to uncertainty in the values of $\left(\eta_{o} L / D\right)_{o}, M_{o}$ and $\left(C_{L}\right)_{o}$. This can be expressed as

$$
\frac{d\left(\eta_{o} L / D\right)}{\left(\eta_{o} L / D\right)}=g_{1} \frac{d\left(\eta_{o} L / D\right)_{o}}{\left(\eta_{o} L / D\right)_{o}}+g_{2} \frac{d M_{o}}{M_{o}}+g_{3} \frac{d\left(C_{L}\right)_{o}}{\left(C_{L}\right)_{o}},
$$

where

$$
\begin{gathered}
g_{1}=\frac{\left(\eta_{o} L / D\right)_{o}}{\left(\eta_{o} L / D\right)} \frac{\partial\left(\eta_{o} L / D\right)}{\partial\left(\eta_{o} L / D\right)_{o}}, \\
g_{2}=\frac{M_{o}}{\left(\eta_{o} L / D\right)} \frac{\partial\left(\eta_{o} L / D\right)}{\partial M_{o}}
\end{gathered}
$$

and

$$
g_{3}=\frac{\left(C_{L}\right)_{o}}{\left(\eta_{o} L / D\right)} \frac{\partial\left(\eta_{o} L / D\right)}{\partial\left(C_{L}\right)_{o}}
$$

To simplify the analysis, the functions $g_{1}, g_{2}$ and $g_{3}$ are derived using the constant Reynolds number functions developed by Poll ${ }^{(1)}$.

For any combination of Mach number and lift coefficient, it can be seen that $\left(\eta_{o} L / D\right)$ is always directly proportional to $\left(\eta_{o} L / D\right)_{o}$ and so $g_{I}$ is constant and equal to unity. However, $g_{2}$ and $g_{3}$ each vary with both $M_{\infty} / M_{o}$ and $C_{L} /\left(C_{L}\right)_{o}$ and the forms of the relationships are given in Figs 12 and 13.

These graphs show that, in general, $\left(\eta_{o} L / D\right)$ exhibits a greater sensitivity to errors in $M_{o}$ than to errors in $\left(C_{L}\right)_{o}$ and that, in the region around the optimum point, the values of $g_{2}$ and $g_{3}$ increase as both $M / M_{o}$ and $C_{L} /\left(C_{L}\right)_{o}$ increase. In practice, for reasons of fuel economy, aircraft tend to operate at Mach numbers between the optimum and the long-range cruise value, $M_{L R C}$; see, for example, Airbus ${ }^{(23)}$, where $M_{L R C}$ is approximately $3.5 \%$ greater than $M_{o}$; see Poll ${ }^{(1)}$. Hence, for normal operating conditions, $\left(M_{\infty} / M_{o}\right)$ lies between 1 and 1.035. However, if there is a need to make up lost time, speeds up to about 1.05 times $M_{o}$ may be used. In addition, all engines have an upper limit on cruise thrust and this imposes upper limits on $C_{L} /\left(C_{L}\right)_{o}$ 


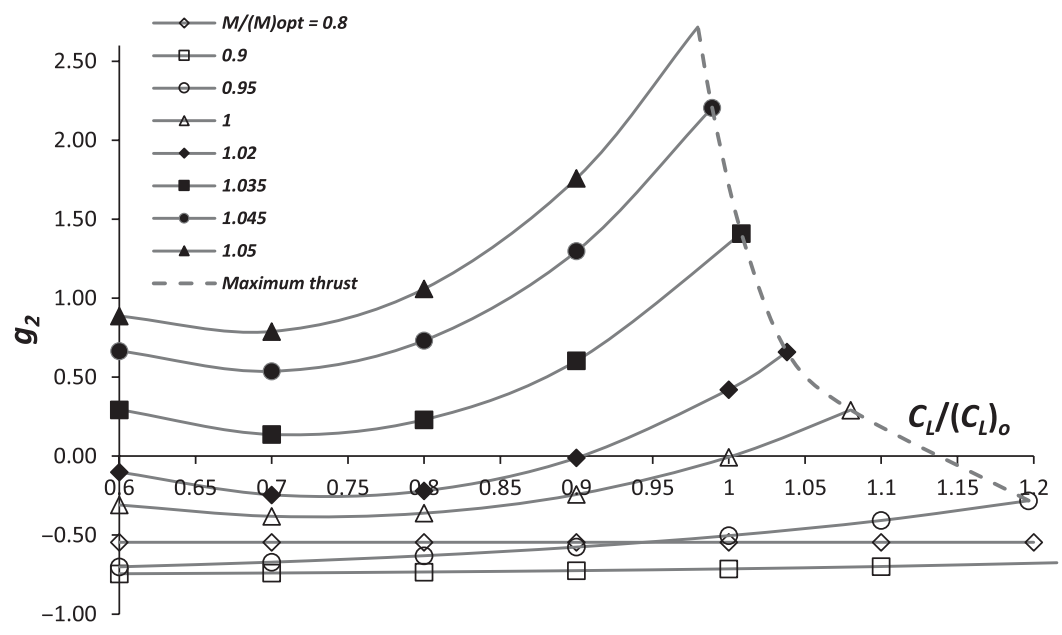

Figure 12. The variation of the function $g_{2}$ with $C_{L} /\left(C_{L}\right)_{0}$ and $M_{\infty} /(M)_{0}$.

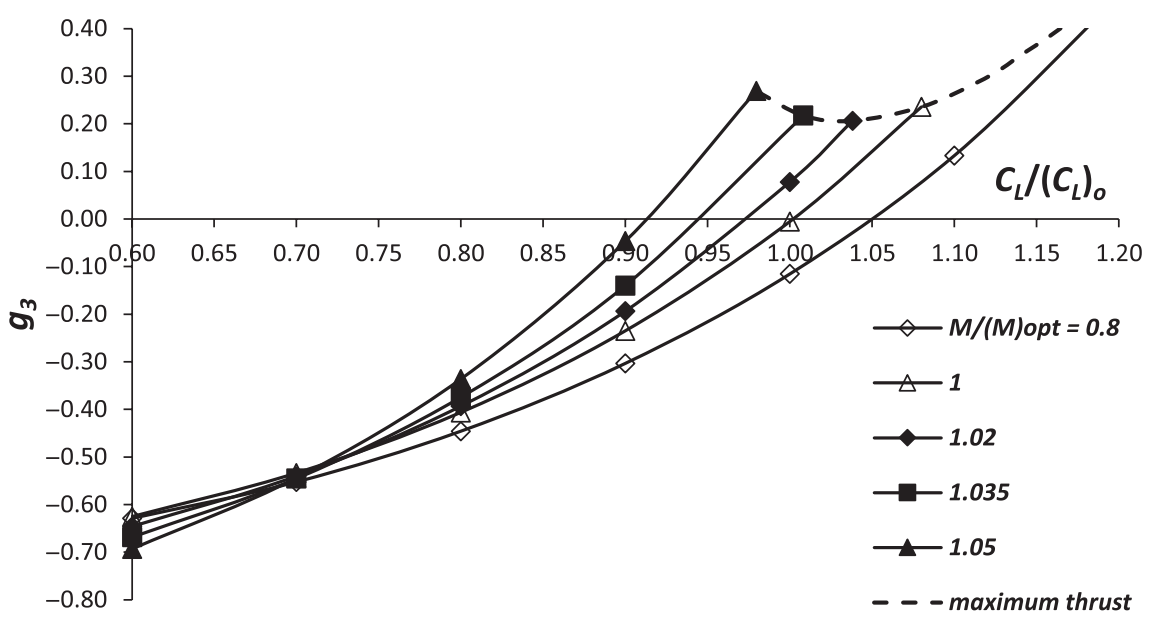

Figure 13. The variation of the function $g_{3}$ with $C_{L} /\left(C_{L}\right)_{0}$ and $M_{\infty} /(M)_{o}$.

for each value of $M / M_{o}$. For a given speed and aircraft weight, the maximum thrust available determines the maximum altitude that can be reached and, under ISA conditions, this is usually about 2,000 to 3,000ft higher than the altitude for minimum fuel burn. In addition, Poll ${ }^{(1)}$ has shown that altitude is linked directly to the term $C_{L} \cdot M_{\infty}{ }^{2}$ and, when the altitude is $2,000 \mathrm{ft}$ higher than the optimum for fuel burn, $C_{L} \cdot M_{\infty}{ }^{2}$ is about 1.08 times $\left(C_{L}\right)_{o} \cdot M_{o}{ }^{2}$. This limiting line has been evaluated for flight in the $I S A^{(15)}$ and is included in the figures ${ }^{10}$.

${ }^{10}$ As shown in Ref. 1, this limiting condition varies with the ambient atmospheric temperature. The colder the air relative to ISA conditions, the greater the attainable lift coefficient at a given Mach number. 
It is clear from the figures that $g_{2}$ only exceeds unity at 'catch up' speeds, whilst $g_{3}$ never exceeds 0.7 at any condition. Therefore, the method has the potential to provide reasonable fuel burn rate estimates. In general, estimates of $\left(\eta_{o} L / D\right)$ exhibit the highest sensitivity to errors in $\left(\eta_{o} L / D\right)_{o}$, since these quantities are directly proportional to one another. Next comes $M_{o}$, where, in general, there is some alleviation of errors, whilst $\left(\eta_{o} L / D\right)$ exhibits least sensitivity to errors in $\left(C_{L}\right)_{o}$.

\subsection{PARAMETER ACCURACY}

Values of $\Psi_{0}$ have been obtained from Equation (23) using the relations set out in Appendix B. Comparisons with a limited amount of flight-test data indicate that the values are accurate to about $\pm 10 \%$. Estimates of $\Psi_{l}$ have been derived from payload-range diagrams contained in the manufactures' APRs. Two independent schemes have been used and the results compared with the limited amount of data available from other sources. Provided that the payload-range diagrams are based upon reliable information, $\Psi_{l}$ is expected to be accurate to approximately $\pm 5 \%$. The Mach number at the optimum condition, $M_{o}$, has been linked to either the longrange cruise Mach number $\left(M_{o}\right.$ equal to $\left.1.035\left(M_{L R C}\right)\right)$ or the $M_{M O}\left(M_{o}\right.$ equal to $\left.0.910\left(M_{M O}\right)\right)$. When $M_{L R C}$ is obtained from either the $A P R$, or the FCOM, the resulting values of $M_{o}$ are expected to be accurate to $\pm 1 \%$. However, if they are estimated from the $M_{M O}$ given in the $T C D S$, the available data suggest that the accuracy drops to about $\pm 4 \%$. Estimates of $e_{o}$ are obtained by first using Equations (24)-(26) to obtain $e_{L S}$, which is converted to $e_{o}$ by using Equation (54) with $C d_{o}$ being set to $\left(C d_{o}\right)_{a v g}$. Overall the values of $e_{L S}$ are accurate to $\pm 8 \%$, with the greatest uncertainty being associated with the parameter $k_{1}$. Similarly, given the observed maximum uncertainty on $\left(C d_{o}\right)_{o}( \pm 10 \%)$ and $\eta_{2}( \pm 15 \%),\left(e_{o} / e_{L S}\right)$ is expected to be accurate to better than $\pm 5 \%$. Based upon the uncertainty in the component parts, $\Psi_{2}$ (Equation (60)) and, hence, $\left(C_{L}\right)_{o}$ (Equation (20)) are believed to be accurate to better than $\pm 10 \%$. Therefore, given the levels of attenuation indicated in Figs 12 and 13, in the aircraft's normal operating range, estimates of $\left(\eta_{o} L / D\right)$ are expected to be in error by no more than $5 \%$ in the majority of cases, with the worst cases being about $10 \%$.

The overall propulsive efficiency of the engine at the optimum condition, $\left(\eta_{o}\right)_{o}$, is estimated from a combination of $\Psi_{1}, \Psi_{3}$ and $\Psi_{4}$. Since $\Psi_{3}$ Equation (61) has the same level of uncertainty as $\Psi_{2}$, i.e. $\pm 10 \%,\left(\eta_{o}\right)_{o}$ is expected to have errors of no more than $\pm 10 \%$. Finally, the thrust at the optimum condition is obtained from the aircraft weight and $\Psi_{3}$. Therefore, assuming that the weight is known, the thrust estimate will have an error of less than $\pm 10 \%$. It is also important to note that the thrust obtained in this way is the 'installed' value; That is to say, it includes the effects of thrust loss due to airframe engine interaction and all the operational power off-take requirements for electrical power generation, air conditioning and de-icing.

\subsection{CONCLUSIONS}

The normalisation scheme proposed by Poll ${ }^{(1)}$ has been developed into a simple, selfcontained, physically complete method for the determination of the cruise fuel burn rate, thrust and engine overall efficiency of a turbofan transport aircraft. However, to determine these quantities for a specific aircraft, values must be attributed to eight constants, which are characteristic of the aircraft and engine combination. In the notation used in this paper, these constants are $\psi_{1}$ to $\psi_{6}, \tau$ and $\eta_{2}$. Some of these constants are simple and the data required 
are readily obtained from information sources that are in the public domain. However, others are complex and require information that is not easily found.

Simple, reliable methods have been developed for the estimation of all the parameters. Where possible, well-established aerodynamic theory has been used. However, for those cases where this has not been possible, empirical methods have been proposed. A table giving a complete set of parameters for some 53 aircraft has been presented. These are the aircraft that are currently responsible for over $85 \%$ of aviation's global greenhouse-gas emissions.

At the present stage of development, it is believed that the estimates of cruise fuel burn rate will be in error by no more than $5 \%$ in the majority of cases and about $10 \%$ in the worst case for the aircraft in the tables. However, this needs to be tested by comparison with other methods currently being used by the environmental science community and this will be addressed in future work.

Finally, an important characteristic of the method is its modular construction. This means that, should more accurate sub-models be developed or better empirical data become available, these can be easily incorporated.

\section{REFERENCES}

1. Poll, D.I.A. On the relationship between non-optimum operations and fuel requirement for large civil transport aircraft with reference to environmental impact and contrail avoidance. The Aeronautical J., 2018, 122, (1258), pp 1827-1870.

2. Poll, D.I.A. and Schumann, U. An estimation method for the fuel burn and other performance characteristics of civil transport aircraft in the cruise Part 1 Fundamental quantities and governing relations for a general atmosphere. The Aeronautical J., 2020, 1-39. doi: 10.1017/aer.2020.62.

3. Schumann, U., Busen, R. and Plohr, M. Experimental test of the influence of propulsion efficiency on contrail formation, J. Aircr., 2000, 37, 1083-1087. doi: 10.2514/2.2715.

4. Shevell, R.S. Fundamentals of Flight, 2nd ed, Prentice Hall International (UK), London, 1989, ISBN 0-13-339060-8.

5. EASA, Airbus A318-A319-A320-A321 Type-Certificate Data Sheet, TCDS A.064 Issue 02, European Aviation Safety Agency, June 2006.

6. Airbus, Aircraft characteristics for airport and maintenance planning. https://www.airbus.com/ aircraft/support-services/airport-operations-and-technical-data/aircraft-characteristics.html

7. JACKSON, P. Jane's All the World's Aircraft 2006/2007, 97th ed, Jane's Information Group, 2006, ISBN 978-0710627452.

8. Daly, M. Jane's Aero-Engines, 2nd ed, HIS, Markit, London, 2014, ISBN 978-0710630926.

9. EASA (2018). ICAO Engine Emissions Databank. https://www.easa.europa.eu/easa-and-you/ environment/icao-aircraft-engine-emissions-databank

10. Jenkinson, L.R., Simpkin, P. and Rhodes, D. Civil Jet Aircraft Design, Arnold, 1999, ISBN 0340 $74152 \mathrm{X}$

11. Torenbeek, E. Synthesis of Subsonic Airplane Design, Kluwer Academic Press, 1988. ISBN 90 24727243

12. TORENBEeK, E. Advanced aircraft design - conceptual design, analysis and optimisation of subsonic civil airplanes, 2013, Chichester, West Sussex, UK: John Wiley and Sons, ISBN 9781119969303.

13. OвеRт, E. Aerodynamic Design of Transport Aircraft, Delft University Press, IOS, The Netherlands, 2009. ISBN 978-1-58603-970-7

14. Poll, D.I.A. Transition in the infinite swept attachment line boundary layer. The Aeronautical Quarterly, 1979, 30, pp 607-628.

15. ICAO (1964), Manual of the ICAO Standard Atmosphere Rep., ICAO Document No. 7488, 2nd ed.

16. ESDU Subsonic lift-dependent drag due to the trailing vortex wake for wings without camber of twist. Engineering Sciences Data Unit Item 74035, October 1974 (amended April 1996). 
17. McLean, D. Understanding Aerodynamics arguing from the real physics, Wiley, Chichester, UK, 2013, 978-1-119-96751-4

18. Shevell, R.S. and BAYAn, F.P. Development of a method for predicting the drag divergence Mach number and the drag due to compressibility for conventional and supercritical wings. SUDAAR 552 (NASA Ames Research Center Grant Number NAG 2-18), Department of Aeronautics and Astronautics, Stanford University, July 1980.

19. Cumpsty, N.A. and Heyes, A.L. Jet Propulsion. Cambridge University Press, 3rd ed, 2015, ISBN 978-1-107-51122-4.

20. ESDU Approximate methods for estimation of cruise range and endurance: aeroplanes with turbo-jet and turbo-fan engines. Engineering Sciences Data Unit Item 73019, October 1973 (amended May 1982).

21. Martinez-Val, R., Palacin, J.F. and Perez, E. The evolution of jet airliners explained through the range equation. Aerospace Engineering, Proc. IMechE., 2008, 222, pp 915-919.

22. Randle, W.E., Hall, C.A. and Vera-Morales, M. Improved range equation based on aircraft data. J. Aircr., 2011, 48, (4), pp 1291-1298.

23. Airbus Getting to grips with aircraft performance. Airbus Customer Services, Toulouse, France, January 2002.

24. Nelder, J.A. and Mead R. A simplex method for function minimization. Computer J., 1965, 7, pp 308-313.

25. Schumann, U., Mayer, B., Graf, K. and Mannstein, H. A parametric radiative forcing model for contrail cirrus, J. Appl. Meteorol. Clim., 2012, 51, 1391-1406, doi: 10.1175/JAMC-D-11-0242.1.

\section{APPENDIX A. A SUMMARY OF THE PRINCIPAL EQUATIONS FROM POLL(1) AND POLL AND SCHUMANN(2).}

At a given Mach number and Reynolds number, i.e. at any specified combination of speed and height,

$$
\frac{\left(\eta_{o} L / D\right)^{R}}{\left(\eta_{o} L / D\right)_{o}^{R}}=\frac{\left(\eta_{o} L / D\right)_{B}^{R}}{\left(\eta_{o} L / D\right)_{o}^{R}}\left(\frac{\left(\eta_{o} L / D\right)^{R}}{\left(\eta_{o} L / D\right)_{B}^{R}}\right)=\text { Function }\left(\frac{C_{L}}{\left(C_{L}\right)_{o}^{R}}, \frac{M_{\infty}}{M_{o}^{R}}\right),
$$

where subscript $B$ (= best) refers to the maximum value of $\left(\eta_{o} L / D\right)$ at a given Mach number, whilst the subscript $o$ refers to the optimum value.

The first term on the right-hand side of the equation is a function of Mach number only, and it was shown in Ref. 1 that this could be adequately approximated by two simple relations. The original relations were found to give problems when used in numerical analyses and so improved versions were developed in Ref. 2 and are used here, i.e. if $0.80<M_{\infty} / M_{o}^{R}<0.99$,

$$
\frac{\left(\eta_{o} L / D\right)_{B}^{R}}{\left(\eta_{o} L / D\right)_{o}^{R}}=f_{1} \approx 1-6.00\left(\frac{M_{\infty}}{M_{o}^{R}}-1\right)^{2}-15.0\left(\frac{M_{\infty}}{M_{o}^{R}}-1\right)^{3}
$$

whilst, if $0.99 \leq M_{\infty} / M_{o}^{R}<1.08$,

$$
\begin{aligned}
f_{1} \approx & 1-5.8965\left(\frac{M_{\infty}}{M_{o}^{R}}-1\right)^{2}+0.36024\left(\frac{M_{\infty}}{M_{o}^{R}}-1\right)^{3} \\
& -31.684\left(\frac{M_{\infty}}{M_{o}^{R}}-1\right)^{4}-53313\left(\frac{M_{\infty}}{M_{o}^{R}}-1\right)^{5}
\end{aligned}
$$


As shown in Ref. 1, the second term on the right-hand side of (A-1) may be approximated by the function

$$
\frac{\left(\eta_{o} L / D\right)^{R}}{\left(\eta_{o} L / D\right)_{B}^{R}} \approx 1+\frac{A}{2}\left(\frac{C_{L}}{\left(C_{L}\right)_{B}^{R}}-1\right)^{2}+\frac{B}{6}\left(\frac{C_{L}}{\left(C_{L}\right)_{B}^{R}}-1\right)^{3}
$$

where the coefficients $A$ and $B$ also depend only upon $M_{\infty} / M_{o}^{R}$, i.e. if $\left(M_{\infty} / M_{o}^{R}\right)<0.975$,

$$
A=B \approx-2.6
$$

otherwise

$$
A \approx-\left(2.6+120\left(\frac{M_{\infty}}{M_{o}^{R}}-0.975\right)^{2}\right)
$$

and

$$
B \approx-\left(2.6+270\left(\frac{M_{\infty}}{M_{o}^{R}}-0.975\right)^{2}\right)
$$

Furthermore,

$$
\frac{\left(C_{L}\right)^{R}}{\left(C_{L}\right)_{B}^{R}}=\left(\frac{\left(C_{L}\right)^{R}}{\left(C_{L}\right)_{o}^{R}}\right) /\left(\frac{\left(C_{L}\right)_{B}^{R}}{\left(C_{L}\right)_{o}^{R}}\right),
$$

where, as shown in Ref. 2, for $0.80<M_{\infty} / M_{o}^{R}<1.08$,

$$
\frac{\left(C_{L}\right)_{B}^{R}}{\left(C_{L}\right)_{o}^{R}}=f_{2} \approx 1.05-14.80\left(\frac{M_{\infty}}{M_{o}^{R}}-0.80\right)^{3}+116.75\left(\frac{M_{\infty}}{M_{o}^{R}}-0.80\right)^{4}-370\left(\frac{M_{\infty}}{M_{o}^{R}}-0.80\right)^{5} .
$$

Finally, since, as argued in Ref. 2,

$$
\begin{gathered}
\left(\eta_{o} L / D\right)_{B}^{R} \approx f_{1}\left(\eta_{o} L / D\right)_{o}^{R}=f_{1} \cdot \psi_{1}\left(\frac{1}{C_{F}^{a c}}\right)^{\left(\frac{1+\tau}{2}\right)}, \\
\left(C_{L}\right)_{B}^{R} \approx f_{2}\left(C_{L}\right)_{o}^{R}=f_{2} \cdot \psi_{2}\left(C_{F}^{a c}\right)^{\left(\frac{1-\tau}{2}\right)}
\end{gathered}
$$

and

$$
M_{o}^{R} \approx \psi_{4}
$$

where $\psi_{1}, \psi_{2}$ and $\psi_{4}$ are known constants for a given aircraft and $C_{F}$ depends only upon Reynolds number - see Equation (7), Equations (A-1) to (A-12) can be used to estimate the value of $\left(\eta_{o} L / D\right)$ at any combination of speed and altitude.

In Ref. 2, this model was used to find the Reynolds number and the Mach number at which $\left(\eta_{o} L / D\right)$ reaches the optimum value for an aircraft of specified mass operating in a completely 
general atmosphere. This requires the introduction of two additional quantities, $\Gamma$ and $\iota$, that capture the effect of temperature variation in the vertical direction. If

$$
\begin{gathered}
\chi=\frac{\left(p_{T P}\right)_{I S A}}{p_{\infty}} \text { and } \phi=\frac{\mu_{\infty} a_{\infty}}{\left(\mu_{T P} a_{T P}\right)_{I S A}} \\
\Gamma=\frac{\chi}{\phi} \frac{d \phi}{d \chi} \approx 277(1-\overline{\Delta T}) L R
\end{gathered}
$$

where

$$
\overline{\Delta T}=\frac{\Delta T}{\left(T_{T P}\right)_{I S A}}=\frac{T_{\infty}}{\left(T_{T P}\right)_{I S A}}-\left(\frac{T_{\infty}}{T_{T P}}\right)_{I S A}
$$

and

$$
L R=\frac{1}{\left(T_{T P}\right)_{I S A}} \frac{d T_{\infty}}{d F L}
$$

The coefficient $\iota$ is defined as

$$
\iota=1+278.8(L R)_{I S A}
$$

Therefore, if the aircraft is flying at an altitude such that $\chi$ is less than or equal to unity, $\iota$ is equal to 0.74505 and, if $\chi$ is greater than unity, it is equal to 1.0 .

The analysis leads to the specification of a number of atmospheric functions, labelled the ' $G$ ' functions. Those relevant to the optimum conditions are

$$
G_{2} \approx\left(\left(1+1.34 \overline{\Delta T}_{o}\right)\left(\left(1+\Delta_{o}\right)(1-\varepsilon)\right)^{i_{o}}(1+\varepsilon)^{\left(2 i_{o}-1\right)}\right)^{-\kappa_{o}},
$$

where

$$
\begin{aligned}
& \varepsilon \approx-0.000260(1+2.825 \tau)\left(1+30.18(1-0.66 \tau) \Gamma_{o}+10.27(1-0.57 \tau) \Gamma_{o}^{2}\right. \\
& \left.+1.91(1-1.78 \tau) \Gamma_{o}^{3}\right), \\
& \quad \Delta_{o} \approx-0.02946(1+0.956 \tau)\left(1+1.14 \Gamma_{o}+0.14 \Gamma_{o}^{2}\right)
\end{aligned}
$$

and

$$
\kappa_{o}=\left(\frac{2}{2-i_{o} b(1-\tau)}\right)
$$

From which it follows that

$$
\begin{gathered}
G_{3}=\left(G_{2}\right)^{-b} \\
G_{4}=\left(f_{2}\right)_{o}\left(1+\Delta_{o}\right)\left(G_{3}\right)^{\left(\frac{1-\tau}{2}\right)}
\end{gathered}
$$


and

$$
G_{5}=\left(f_{1}\right)_{o}\left(1+\frac{A_{o}}{2} \Delta_{o}^{2}+\frac{B_{o}}{6} \Delta_{o}^{3}\right)\left(\frac{1}{G_{3}}\right)^{\left(\frac{1+\tau}{2}\right)}
$$

Finally, two additional ' $f$ ' functions are introduced as

$$
f_{3} \approx f_{1}\left(\left(\frac{1+1.34 \overline{\Delta T}_{o}}{1+1.34 \overline{\Delta T}}\right) \chi_{o}^{\left(i_{o}-i\right)}\left(\frac{M_{\infty}}{(1+\varepsilon) M_{o}^{R}}\right)^{(1-2 i)}\right)^{b\left(\frac{1+\tau}{2}\right)}
$$

and

$$
f_{4} \approx \frac{\left(1+\Delta_{o}-\varepsilon\right)}{f_{2}}\left(\left(\frac{1+1.34 \overline{\Delta T}_{o}}{1+1.34 \overline{\Delta T}}\right) \chi_{o}^{\left(i_{o}-i\right)}\left(\frac{M_{\infty}}{(1+\varepsilon) M_{o}^{R}}\right)^{(1-2 i)}\right)^{b\left(\frac{1-\tau}{2}\right)},
$$

where

$$
v=1-i b\left(\frac{1-\tau}{2}\right)
$$

\section{APPENDIX B. ESTIMATION OF $\left(\mathrm{Cd}_{\mathrm{O}} / \mathrm{C}_{F}\right)$ FROM THE AIRCRAFT EXTERNAL GEOMETRY}

\section{a. Fuselage}

The fuselage slenderness ratio, $\lambda_{f}$, is the ratio of the fuselage total length, $l_{f}$, to the fuselage mean diameter, $d_{f}$. Since civil transport aircraft usually have a passenger cabin with a constant width, $b_{f}$, and a constant height, $h_{f}$, the mean diameter is defined, on the basis of bodies of equal cross-sectional area, as

$$
d_{f}=b_{f}\left(\frac{h_{f}}{b_{f}}\right)^{1 / 2}
$$

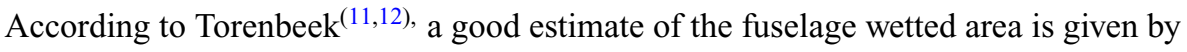

$$
\frac{\left(S_{f}\right)_{w e t}}{S_{r e f}} \approx \pi\left(1-\frac{2}{\lambda_{f}}\right)^{2 / 3}\left(1+\frac{1}{\lambda_{f}^{2}}\right) \frac{l_{f} d_{f}}{S_{r e f}}
$$

and, to maintain consistency, the Torenbeek value of the fuselage form factor, $\left(F_{F}\right)_{f}$ is used, where

$$
\left(F_{F}\right)_{f} \approx\left(1+\frac{2.2}{\lambda_{f}^{1.5}}+\frac{3.8}{\lambda_{f}^{3}}\right)
$$


However, since Torenbeek does not provide values for the interference and secondary drag factors, $\left(F_{I}\right)_{f}$ and $\left(F_{S}\right)_{f}$, the values proposed by Jenkinson el al. ${ }^{(13)}$ are used, i.e.

$$
\left(F_{I} F_{S}\right)_{f} \approx 1.1
$$

From Equation (7) of the main text, the ratio of the mean skin friction coefficient for the fuselage to the reference mean skin friction coefficient is

$$
\frac{\left(C_{F}\right)_{f}}{\left(C_{F}\right)_{a c}} \approx\left(\frac{l_{a c}}{l_{f}}\right)^{0.14} .
$$

b. Wing, horizontal tail, vertical tail and engine nacelles

The wetted areas of the wing, horizontal tail, vertical tail and nacelles are measured directly from the three-view, general arrangement drawings given in the airport planning document, whilst the various form factors, interference factors and secondary drag factors are again taken from Jenkinson el al. ${ }^{(13)}$.

For the wing

$$
\left(F_{F}\right)_{w} \approx\left(1+2.9(t / c)_{w} \cos ^{2}\left(\Lambda_{w}\right)\right),\left(F_{I} F_{S}\right)_{w} \approx 1.06
$$

and

$$
\frac{\left(C_{F}\right)_{w}}{\left(C_{F}\right)_{a c}} \approx\left(\frac{l_{a c}}{l_{w}}\right)^{0.14},
$$

where $l_{w}$ is the mean wing chord, defined as the wing reference area, $S_{\text {ref }}$, divided by the wingspan. For the horizontal tail (tailplane),

$$
\left(F_{F}\right)_{h t} \approx 1+3.5(t / c)_{h t} \approx 1.20, \quad\left(F_{I} F_{S}\right)_{h t} \approx 1.20
$$

and

$$
\frac{\left(C_{F}\right)_{h t}}{\left(C_{F}\right)_{a c}} \approx\left(\frac{l_{a c}}{l_{h t}}\right)^{0.14}
$$

where $l_{h t}$ is the mean chord length, defined as the horizontal-tail plan area divided by its span. Similarly, for the vertical tail,

$$
\left(F_{F}\right)_{v t} \approx 1+3.5(t / c)_{v t} \approx 1.18, \quad\left(F_{I} F_{S}\right)_{v t} \approx 1.20
$$

and

$$
\frac{\left(C_{F}\right)_{v t}}{\left(C_{F}\right)_{a c}} \approx\left(\frac{l_{a c}}{l_{v t}}\right)^{0.14},
$$

where $l_{v t}$ is the mean chord length, defined as the vertical-tail (fin) plan area divided by the fin height. 
For the nacelles,

$$
\left(F_{F} F_{I} F_{S}\right)_{n a c} \approx 1.44
$$

and

$$
\frac{\left(C_{F}\right)_{n a c}}{\left(C_{F}\right)_{a c}} \approx\left(\frac{l_{a c}}{l_{n a c}}\right)^{0.14}
$$

where $l_{\text {nac }}$ is the length of the nacelle. Finally, for best agreement with the flight data from Ref. 13,

$$
F_{o}=0.98
$$

\section{APPENDIX C. ESTIMATION OF THE RESERVE FUEL REQUIREMENT}

Under the European Joint Aviation Regulations, formerly the JAR-OPS 1 and now the EU Air OPS regulatory requirements (see Airbus ${ }^{(23)}$ ), the minimum reserve fuel has five elements:

a) 'contingency fuel', $M F_{\text {cont }}$, at $5 \%$ of the trip fuel mass, $T F M$

b) 'additional fuel', $M F_{a d}$, sufficient to fly for $15 \mathrm{~min}$ at the holding speed at a height of $1,500 \mathrm{ft}$ above the destination airport

c) 'alternate fuel', $M F_{\text {alt }}$, enough to fly about $200 \mathrm{~nm}(\approx 370 \mathrm{~km})$ from the original destination to an alternate airport

d) 'final reserve fuel', $M F_{f r}$, sufficient to fly for $30 \mathrm{~min}$ at the holding speed at a height of $1,500 \mathrm{ft}$ above the alternate airport and

e) 'extra fuel', $M F_{\text {ext }}$,

The US Federal Aviation Regulations (FARs) are similar.

Elements (a) and (b) apply to the basic journey from departure to the intended destination. The contingency fuel is a straight $5 \%$ increase in fuel load, whilst the 'additional' fuel requirement is based upon a fixed time spent in a holding pattern near the destination airport. However, for a given aircraft, this holding fuel can always be equated to the amount required for an equivalent, additional cruise distance, $R_{a d}$, in which case

$$
\frac{(M F)_{c o n t}}{T O M}+\frac{(M F)_{a d}}{T O M} \approx 0.05+\left(0.95-E X P\left(-\frac{X_{a d}}{n}\right)\right)\left(1-\alpha_{t}\right) .
$$

The diversion to the alternate airport begins with a missed approach at the destination. Therefore, to a good approximation, the diversion is the same as a normal trip from the destination to the alternate, taking-off at the planned landing mass, $L M$. Hence, if the distance to the alternate airport is $R_{d a}$,

$$
\frac{(M F)_{a l t}}{T O M} \approx \alpha_{a l t} \frac{L M}{T O M} \approx\left(1-\operatorname{EXP}\left(-\left(\left(\varepsilon_{c d}\right)_{\min }+\frac{X_{d a}}{n}\right)\right)\right)\left(1-\alpha_{t}\right) .
$$


The final reserve fuel requirement is based upon a fixed time spent in a holding pattern near the alternate airport. However, once again, for a given aircraft, this holding fuel can be equated to an equivalent, extra diversion distance, $R_{f}$, in which case,

$$
\frac{(M F)_{a l t}}{T O M}+\frac{(M F)_{f r}}{T O M} \approx\left(1-\operatorname{EXP}\left(-\left(\left(\varepsilon_{c d}\right)_{\min }+\frac{\left(X_{d a}+X_{f r}\right)}{n}\right)\right)\right)\left(1-\alpha_{t}\right) .
$$

Finally, the 'extra' fuel component is specified either by the operator, or the captain. This is a matter of judgement and this element is not subject to any regulatory rules. However, as with the other elements, this 'extra' fuel can always be expressed as an additional distance flown.

The total minimum reserve fuel is obtained by combining Equations (C-1), (C-2) and (C-3). Moreover, since $\left(\varepsilon_{c d}\right)_{\min }, X_{a d}, X_{d a}$ and $X_{f r}$ are always very small compared with unity,

$$
\frac{\left(M F_{r e s}\right)_{\min }}{T O M}=\beta_{\text {min }} \approx 0.05 \alpha_{t}+\left(\left(\varepsilon_{c d}\right)_{\min }+\frac{\left(X_{a d}+X_{d a}+X_{f r}\right)}{n}\right)\left(1-\alpha_{t}\right) .
$$

For any given aircraft, if the rules governing the reserve fuel and the 'extra' fuel are known, the 'distances' $R_{a d}, R_{d a}$ and $R_{f r}$ can be computed. However, whatever the rules and whatever 'extra' fuel is carried, it follows from Equation (54) that

$$
\beta_{\min } \approx 0.05 \alpha_{t}+\lambda\left(1-\alpha_{t}\right)=\frac{(0.05+(\lambda-0.05)(Z F M / T O M))}{(1.05-\lambda)}
$$

where $\lambda$ is a constant.

\section{APPENDIX D. METHOD 2 FOR THE DETERMINATION OF $\left(\eta_{0} L / D\right)_{a v g}$}

In order to find the $\left(\eta_{o} L / D\right)_{a v g}$ and $\lambda$ solution pairs, Equation (75) is re-written as

$$
\left(\eta_{o} L / D\right)_{a v g}=\frac{\left(-g R_{t} / L C V\right)}{L N\left(E X P\left(\left(\varepsilon_{c d}\right)_{\min }\right)(0.05+Z F M / T O M) /(1.05-\lambda)\right)},
$$

where $\left(\varepsilon_{c d}\right)_{\min }$ has been taken to be 0.0067 . Equation (D-1) has to be solved numerically for a number of range and zero-fuel mass data pairs, $i=1, \ldots, N$.

When $N$ equals 2 , there are two equations for the two unknowns, and provided that the data represent two clearly separated payload cases, there is a unique solution. This can be obtained by a Newton iteration based on the linearised version of

$$
f\left(\left(\eta_{o} L / D\right)_{a v g}, \lambda, \frac{g R_{t}}{L C V}, \frac{Z F M}{T O M}\right)=\left(\eta_{o} L / D\right)_{a v g}+\frac{g R_{t} / L C V}{L N\left(1.0067 \frac{(0.05+Z F M / T O M)}{(1.05-\lambda)}\right)}=0 .
$$


Starting with the initial estimates $\lambda_{0}=0$ and

$$
\left(\left(\eta_{o} L / D\right)_{a v g}\right)_{0}=\frac{-\left(\left(\left(g R_{t} / L C V\right)_{1}+\left(g R_{t} / L C V\right)_{2}\right) / 2\right)}{L N\left(1.0067\left(\frac{\left(0.05+\left(\left((Z F M / T O M)_{1}+(Z F M / T O M)_{2}\right) / 2\right)\right)}{1.05}\right)\right)}
$$

the following functions are computed for $i=1$ and 2

$$
\begin{gathered}
f_{0}^{i}=f\left(\left(\left(\eta_{o} L / D\right)_{\text {avg }}\right)_{0}, \lambda_{0},\left(\frac{g R_{t}}{L C V}\right)_{i},\left(\frac{Z F M}{T O M}\right)_{i}\right), \\
\frac{\partial f_{0}^{i}}{\partial\left(\eta_{o} L / D\right)_{\text {avg }}}=1
\end{gathered}
$$

and

$$
\frac{\partial f_{0}^{i}}{\partial \lambda}=\frac{\left(g R_{t} / L C V\right)_{i}}{\left(L N\left(1.0067\left(\frac{\left(0.05+(Z F M / T O M)_{i}\right)}{1.05-\lambda_{0}}\right)\right)\right)^{2}\left(\lambda_{0}-1.05\right)} .
$$

Improved solutions are then obtained with the help of a determinate, $D$, of the linear system of equations, namely

$$
\begin{gathered}
D=\frac{\partial f_{0}^{2}}{\partial \lambda}-\frac{\partial f_{0}^{1}}{\partial \lambda}, \\
\left(\eta_{o} L / D\right)_{\text {avg }}=\left(\left(\eta_{o} L / D\right)_{\text {avg }}\right)_{0}+\frac{f_{0}^{2} \frac{\partial f_{0}^{1}}{\partial \lambda}-f_{0}^{1} \frac{\partial f_{0}^{2}}{\partial \lambda}}{D}
\end{gathered}
$$

and

$$
\lambda=\lambda_{0}+\frac{f_{0}^{1}-f_{0}^{2}}{D}
$$

After replacing $\left.\left(\left(\eta_{o} L / D\right)_{a v g}\right)_{0}, \lambda_{0}\right)$ by $\left(\left(\eta_{o} L / D\right)_{a v g}, \lambda\right)$, the computation can be refined iteratively to obtain the final values.

When $N$ exceeds 2, the system of equations is overdetermined and the 'solution' is obtained from a standard least-squares fit. Since, $\left(\eta_{o} L / D\right)_{a v g}$ can be determined from Equation (D-1), $\lambda$ is varied systematically until the results provide an optimal fit in a suitable quadratic error norm. First estimates are obtained by assuming that $\lambda$ equals 0.05 , and subsequently, the results are refined using either the simplex method of Nelder and $\mathrm{Mead}^{(24)}$ or a robust systematic search algorithm, e.g. Schumann et al. ${ }^{(25)}$. A solution with a precision better than $1.0 \mathrm{E}-5$ is obtained typically after 30 evaluations of (D-1). 